\title{
Dominating Energy in Neutrosophic graphs
}

\author{
M. Mullai ${ }^{1 *}$, Said Broumi ${ }^{2}$ \\ ${ }^{1}$ Department of Mathematics(DDE), Alagappa University, Karaikudi, Tamilnadu, India. \\ ${ }^{2}$ Laboratory of Information processing, Faculty of Sciences, Ben M'SiK, University Hassan II,B.P \\ 7955, Sidi Othman, Casablanca, Morocco. \\ ${ }^{1}$ mullaim@alagappauniversity.ac.in, ${ }^{2}$ broumisaid78@gmail.com
}

\begin{abstract}
Dominating energy of graphs plays a vital role in the field of application in energy. Results by applying neutrosophic graph theory is more efficient than other existing methods. So, dominating energy of neutrosophic graphs will also give more accurate results than other exixting methods in the field of energy. This article introduces dominating energy of neutrosophic graphs. Dominating energy of a neutrosophic graph, dominating neutrosophic adjacency matrix, eigen values for the dominating energy of a neutrosophic graphs and complement of neutrosophic graphs are defined with examples. Also, dominating energy in union and join operations of neutrosophic graphs are developed and some theorems in dominating energy of a neutrosophic graphs are derived here.
\end{abstract}

Keywords: Neutrosophic graph, dominating energy of neutrosophic graph, eigen values for dominating energy of a neutrosophic graph.

\section{Introduction}

Fuzzy set plays a vital role in the area of interdisciplinary research. Fuzzy graph relation was introduce by Zadeh[20] and it has many real world applications. Rosenfield[12] used fuzzy relations on fuzzy sets and derived the structure of fuzzy graphs.

Recently, intuitionistic fuzzy set area takes important rule from normal mathematics to computer sciences, information sciences and communications systems. Spectrum of graphs is used in statistical physics problems and in combinatorial optimization problems. Spectrum of a graph also plays an important role in pattern recognition, virus propagation in computer networks and in secure datas in databases. The spectrum of a graph is used in the field of of energy.

Let $d_{i}$ be the degree of $i^{\text {th }}$ vertex of $G, i=1,2, \ldots ., n$. The spectrum of graph $G$ consisting of $\lambda_{1}, \lambda_{2}, \ldots \ldots ., \lambda_{n}$ is the spectrum of its adjacency matrix[3]. The Laplacian spectrum of the graph $G$ consisting of $\mu_{1}, \mu_{2}, \ldots \ldots . ., \mu_{n}$ is the spectrum of its Laplacian matrix.

The following relations are satisfied by ordinary and laplacian graph eigen values.

$$
\begin{aligned}
& \sum_{\mathrm{i}=1}^{\mathrm{n}} \lambda_{\mathrm{i}}=0, \sum_{\mathrm{i}=1}^{\mathrm{n}} \lambda_{\mathrm{i}}^{2}=2 \mathrm{~m}, \sum_{\mathrm{i}=1}^{\mathrm{n}} \mu_{\mathrm{i}}=2 \mathrm{~m}, \\
& \sum_{\mathrm{i}=1}^{\mathrm{n}} \mu_{\mathrm{i}}^{2}=2 \mathrm{~m}+\sum_{\mathrm{i}=1}^{\mathrm{n}} \mathrm{d}_{\mathrm{i}}^{2}
\end{aligned}
$$

The study of domination in graphs was Started in 1960,. C.F.De jaenisch[2] tried to find the minimum number of queens required to cover a $\mathrm{n} \times \mathrm{n}$ chess board in 1862 , The independent domination number in graphs was established by Cockayne[1]. Domination in graphs has many applications in several fields. A.Somasundaram and 
S.Somasundaram[17] introduced domination in fuzzy graph in terms of effective edges. Domination using strong arcs was introduced by A Nagoorgani and V.T.Chandrasekaran[4]. R.Parvathi and G.Thamizhenthi[6] developed dominating sets, dominating number, independent set, total dominating and total dominating number in intuitionistic fuzzy graphs. In[19] Vijayragavan et.al developed the dominating energy in products of intuitionistic fuzzy graph. Many authors introduced various concepts and their applications of neutrosophic theory in $[3,5,7,9,10,11,14,15,16,18,22,23,24,25,26,27,28]$

Domination in Neutrosophic graphs are more convenient than fuzzy and intuitionistic fuzzy graphs, which is useful in the field of traffic and communication systems, because the neutrosophic set is a generalization of fuzzy and intuitionistic fuzzy sets. Also neutrosophic concept plays an important role in real world applications when uncertainty and inderminacy occur. The results obtained by using neutrosophics sets are more accurate than fuzzy and intuitionistic fuzzy sets. Dominations in neutrosophic graphs was introduced by M.Mullai [21].

The energy of a graph is used in quantum theory by relating edge of a graph with electron energy of a class of molecule and many applications in the field of energy. Similarly energy of fuzzy graphs and intuitionistic fuzzy graphs are applied in many fields. Dominating energy is more efficient in the field of energy. Compared to dominating energy of fuzzy graphs and intuitionistic fuzzy graphs, dominating energy of neutrosophic graphs is more efficient by giving accurate results in various real life applications. Before analyzing these concepts, dominating energy of neutrosophic graph and dominating energy of different operations of neutrosophic graph are defined with examples and some theorems in dominating energy of neutrosophic graph are established and various results are discussed in this article.

\section{Preliminaries}

This part includes some basic definitions and results in domination theory of graphs that is very helpful to the proposed research work.

Definition 2.1. [5]An intuitionistic fuzzy graph is defined as $G=(V, E, \mu, \gamma)$, where $V$ is the set of vertices and $E$ is the set of edges, $\mu$ is a fuzzy membership function defined on $\mathrm{V} \times \mathrm{V}$ and $\gamma$ is a fuzzy non membership function. Define $\mu\left(v_{i}, v_{j}\right)$ by $\mu_{i j}$ and $\gamma\left(v_{i}, v_{j}\right)$ by $\gamma_{i j}$ such that

$1.0 \leq \mu_{\mathrm{ij}}+\gamma_{\mathrm{ij}} \leq 1$

$2.0 \leq \mu_{\mathrm{ij}}, \gamma_{\mathrm{ij}}, \pi_{\mathrm{ij}} \leq 1$, where $\pi_{\mathrm{ij}}=1-\mu_{\mathrm{ij}}-\gamma_{\mathrm{ij}}$.

Hence, $(V \times V, \mu, \gamma)$ is an intuitionistic fuzzy graph.

Definition 2.2. [8] An intuitionistic fuzzy graph is of the form $G=(V, E)$, where

(i) $\mathrm{V}=\left\{\mathrm{v}_{1}, \mathrm{v}_{2}, \ldots \mathrm{v}_{\mathrm{n}}\right\}$ such that $\mu: \mathrm{V} \rightarrow[0,1], \gamma: \mathrm{V} \rightarrow[0,1]$ denote the degree of membership and nonmembership of the element $v \in V$ respectively and $0 \leq \mu_{1}\left(v_{i}\right)+\gamma_{1}\left(v_{i}\right) \leq 1$ for every $v_{i} \in V_{i}(i=1,2,3, \ldots)$

(ii) $\mathrm{E} \subseteq \mathrm{V} \times \mathrm{V}$ where $\mu_{2}: \mathrm{V} \times \mathrm{V} \rightarrow[0,1]$ and $\gamma_{2}: \mathrm{V} \times \mathrm{V} \rightarrow[0,1]$ are suchthat

$$
\mu_{2}\left(v_{i}, v_{j}\right) \leq \mu_{1}\left(v_{i}\right) \wedge \mu_{1}\left(v_{j}\right)
$$

$$
\begin{aligned}
& \gamma_{2}\left(v_{i}, v_{j}\right) \leq \gamma_{1}\left(v_{i}\right) \wedge \gamma_{1}\left(v_{j}\right) \text { and } \\
& 0 \leq \mu_{2}\left(v_{i}, v_{j}\right)+\gamma_{2}\left(v_{i}, v_{j}\right) \leq 1
\end{aligned}
$$

Definition 2.3. [8] An $\operatorname{arc}\left(v_{i}, v_{j}\right)$ of an intuitionistic fuzzy graph $G$ is called a strong arc if 


$$
\mu_{2}\left(v_{i}, v_{j}\right) \leq \mu_{1}\left(v_{i}\right) \wedge \mu_{1}\left(v_{j}\right) \text { and } \gamma_{2}\left(v_{i}, v_{j}\right) \leq \gamma_{1}\left(v_{i}\right) \wedge \gamma_{1}\left(v_{j}\right)
$$

Definition 2.4. [8] Let $G=\left(V, E, \mu, \gamma, \mu_{1}, \gamma_{1}\right)$ be a dominating intuitionistic fuzzy graph. A dominating intuitionistic fuzzy adjacency matrix $D(G)=\left[d_{i j}\right]$, where

$$
d_{i j}= \begin{cases}\left(\mu_{i j}, \gamma_{i j}\right) & \text { if }\left(v_{i}, v_{j}\right) \in E \\ (1,1) & \text { if } i=j \text { and } v_{i} \in D \\ (0,0) & \text { otherwise }\end{cases}
$$

This dominating intuitionistic fuzzy graph adjacency matrix $D(G)$ can be written as $D(G)=$ $\left(\mu_{D}(G), \gamma_{D}(G)\right)$ where

$$
\mu_{D}(G)= \begin{cases}\mu_{i j} & \text { if }\left(v_{i}, v_{j}\right) \in E \\ 1 & \text { if } i=j \text { and } v_{i} \in D \\ 0 & \text { otherwise }\end{cases}
$$

and

$$
\gamma_{D}(G)= \begin{cases}\gamma_{i j} & \text { if }\left(v_{i}, v_{j}\right) \in E \\ 1 & \text { if } i=j \text { and } v_{i} \in D \\ 0 & \text { otherwise }\end{cases}
$$

Definition 2.5. [8] The eigen values of dominating intuitionistic fuzzy adjacency matrix $D(G)$ is defined as $(X, Y)$ where $X$ is the set of eigen values of $\mu_{D}(G)$ and $Y$ is the set of eigen values of $\gamma_{D}(G)$. The energy of a dominating intuitionistic fuzzy graph $G=\left(V, E, \mu, \gamma, \mu_{1}, \gamma_{1}\right)$ is defined $\left(\sum_{\lambda_{i} \in X}\left|\lambda_{i}\right|, \sum_{\delta_{i} \in Y}\left|\delta_{i}\right|\right)$ where $\sum_{\lambda_{i} \in X}\left|\lambda_{i}\right|$ is the sum of the absolute values of the eigen values of $\mu_{D}(G)$ and it is denoted by the energy of the membership matrix $E\left(\mu_{D}(G)\right)$ and $\sum_{\delta_{i} \in Y}\left|\delta_{i}\right|$ is the sum of the absolute values of the eigen values of $\gamma_{D}(G)$ and it is denoted by the energy of the membership matrix $\mathrm{E}\left(\gamma_{\mathrm{D}}(\mathrm{G})\right)$.

Definition 2.6. [19] Let $\mathrm{G}_{1}=\left\langle\mathrm{V}_{1}, \mathrm{E}_{1}\right\rangle$ and $\mathrm{G}_{2}=\left\langle\mathrm{V}_{2}, \mathrm{E}_{2}\right\rangle$ be two intuitionistic fuzzy graphs with $\mathrm{V}_{1} \cap \mathrm{V}_{2}=\emptyset$ and $G=G_{1} \cup G_{2}=\left\langle V_{1} \cup V_{2}, E_{1} \cup E_{2}\right\rangle$ be the union of $G_{1}$ and $G_{2}$. Then the union of intuitionistic fuzzy graphs $G_{1}$ and $\mathrm{G}_{2}$ is an intuitionistic fuzzy graph defined by

$$
\begin{aligned}
& \left(\mu_{1} \cup \mu_{1}\right)(v)= \begin{cases}\mu_{1}(v) & \text { if } v \in v_{1}-v_{2} \\
\mu_{1}(v) & \text { if } v \in v_{2}-v_{1}\end{cases} \\
& \left(\gamma_{1} \cup \gamma_{1},\right)(v)=\left\{\begin{array}{ll}
\gamma_{1}(v) & \text { if } \quad v \in v_{1}-v_{2} \\
\gamma_{1},(v) & \text { if } \quad v \in v_{2}-v_{1}
\end{array}\right. \text { and } \\
& \left(\mu_{2} \cup \mu_{2 \prime}\right)\left(v_{i}, v_{j}\right)=\left\{\begin{array}{lll}
\mu_{2}\left(e_{i j}\right) & \text { if } & e_{i j} \in E_{1}-E_{2} \\
\mu_{2 \prime}\left(e_{i j}\right) & \text { if } & e_{i j} \in E_{2}-E_{1}
\end{array}\right.
\end{aligned}
$$

where $\left(\mu_{1}, \gamma_{1}\right)$ and $\left(\mu_{1}, \gamma_{1},\right)$ refer the vertex membership and non-membership of $G_{1}$ and $G_{2}$ respectively, $\left(\mu_{2}, \gamma_{2}\right)$ and $\left(\mu_{2}, \gamma_{2}\right)$ refer the edge membership and non-membership of $G_{1}$ and $G_{2}$ respectively.

Definition 2.7. [19] The join of two intutionistic fuzzy graphs

$$
\begin{aligned}
& G=G_{1}+G_{2}=\left\langle V_{1} \cup V_{2}, E_{1} \cup E_{2}\right\rangle \text { defined by } \\
& \left(\mu_{1}+\mu_{1}\right)(v)=\left(\mu_{1} \cup \mu_{1}\right) \text { if } v \in V_{1} \cup V_{2} \\
& \left(\gamma_{1}+\gamma_{1}\right)(v)=\left(\gamma_{1} \cup \gamma_{1}\right)(v) \text { if } v \in V_{1} \cup V_{2}
\end{aligned}
$$


$\left(\mu_{2}+\mu_{2 \prime}\right)\left(v_{i} v_{j}\right)=\left(\mu_{2} \cup \mu_{2 \prime}\right)\left(v_{i} v_{j}\right)$ if $v_{i} v_{j} \in E_{1} \cup E_{2}$

Definition 2.8. [19] The $\alpha$-product of two intuitionistic fuzzy graphs $G_{1}=\left(V_{1}, E_{1}\right)$ and

$G_{2}=\left(V_{2}, E_{2}\right)$ denoted by $G_{1} \odot G_{2}$ is an intuitionistic fuzzy graph

$\mathrm{G}=\left(\mathrm{V}, \mathrm{E},\left\langle\mu_{\mathrm{r}}, \mathrm{v}_{\mathrm{r}}\right\rangle,\left\langle\mu_{\mathrm{rs}}, \mathrm{v}_{\mathrm{rs}}\right\rangle\right)$ where

$1 . V=v_{i} u_{p}$ for all $v_{i} \in V_{1}$ and $u_{p} \in V_{2},\left(V_{1} \cap V_{2}\right)=\emptyset, i=1,2,3, \ldots m, p=1,2,3, \ldots . n$

2.E $=\left\langle v_{\mathrm{i}} \mathrm{u}_{\mathrm{p}}, \mathrm{v}_{\mathrm{j}} \mathrm{u}_{\mathrm{q}}\right\rangle$ such that either one of the following is true:

(i) $\left(\mathrm{v}_{\mathrm{i}}, \mathrm{v}_{\mathrm{j}}\right) \in \mathrm{E}_{1}$ and $\left(\mathrm{u}_{\mathrm{p}}, \mathrm{u}_{\mathrm{q}}\right) \notin \mathrm{E}_{2}$

(ii) $\left(\mathrm{u}_{\mathrm{p}}, \mathrm{u}_{\mathrm{q}}\right) \in \mathrm{E}_{2}$ and $\left(\mathrm{v}_{\mathrm{i}}, \mathrm{v}_{\mathrm{j}}\right) \notin \mathrm{E}_{1}$

3. $\left\langle\mu_{\mathrm{r}}, \mathrm{v}_{\mathrm{r}}\right\rangle$ denote the degrees of membership and non-membership of

vertices of G,and is given by $\left\langle\mu_{r}, v_{r}\right\rangle=\left\langle\min \left(\mu_{i}, \mu_{p}\right), \max \left(v_{i}, v_{p}\right)\right\rangle$ for all

$\mathrm{v}_{\mathrm{r}} \in \mathrm{V}, \mathrm{r}=1,2,3, \ldots \mathrm{m}, \mathrm{n}$

4. $\left\langle\mu_{\mathrm{rs}}, \mathrm{v}_{\mathrm{rs}}\right\rangle$ denote the degrees of membership and non-membership of edges of $\mathrm{G}$, and is given by

$$
\left\langle\mu_{r s}, v_{r s}\right\rangle=\left\{\begin{array}{lll}
\left\langle\min \left(\mu_{i}, \mu_{j}, \mu_{p}\right), \max \left(v_{i}, v_{j}, v_{p}\right)\right\rangle & \text { if }\left(v_{i}, v_{j}\right) \notin E_{1} \text { and } \quad\left(u_{p}, u_{q}\right) \in E_{2} \\
\left\langle\min \left(\mu_{p}, \mu_{j}, \mu_{i j}\right), \max \left(v_{p}, v_{q}, v_{i j}\right)\right\rangle & \text { if }\left(v_{i}, v_{j}\right) \in E_{1} \text { and } \quad\left(u_{p}, u_{q}\right) \notin E_{2} \\
\langle 0,0\rangle & \text { if }\left(v_{i}, v_{j}\right) \in E_{1} \text { and } \quad\left(u_{p}, u_{q}\right) \in E_{2}
\end{array}\right.
$$

Definition 2.9. [19] The $\beta$-product of two intuitionistic fuzzy graphs $G_{1}=\left(V_{1}, E_{1}\right)$ and

$\mathrm{G}_{2}=\left(\mathrm{V}_{2}, \mathrm{E}_{2}\right)$ denoted by $\mathrm{G}_{1} * \mathrm{G}_{2}$ is an intuitionistic fuzzy graph

$\mathrm{G}=\left(\mathrm{V}, \mathrm{E},\left\langle\mu_{\mathrm{r}}, \mathrm{v}_{\mathrm{r}}\right\rangle,\left\langle\mu_{\mathrm{rs}}, \mathrm{v}_{\mathrm{rs}}\right\rangle\right)$ where

$1 . V=v_{i} u_{p}$ for all $v_{i} \in V_{1}$ and $\left.u_{p} \in V_{2}, V_{1} \cap V_{2}\right)=\emptyset, i=1,2,3, \ldots m, p=1,2,3, \ldots . n$

2.E $=\left\langle v_{i} u_{p}, v_{j} u_{q}\right\rangle$ such that either one of the following is true:

(i) $\left(v_{i}, v_{j}\right) \in E_{1}$ when $p \neq q, i \neq j$

(ii) $\left(\mathrm{u}_{\mathrm{p}}, \mathrm{u}_{\mathrm{q}}\right) \in \mathrm{E}_{2}$ when $\mathrm{i} \neq \mathrm{j}, \mathrm{p} \neq \mathrm{q}$

3. $\left\langle\mu_{r}, v_{r}\right\rangle$ denote the degrees of membership and non-membership of vertices of $\mathrm{G}$, and is given by $\left\langle\mu_{\mathrm{r}}, \mathrm{v}_{\mathrm{r}}\right\rangle=\left\langle\min \left(\mu_{\mathrm{i}}, \mu_{\mathrm{p}}\right), \max \left(\mathrm{v}_{\mathrm{i}}, \mathrm{v}_{\mathrm{p}}\right)\right\rangle$ for all

$\mathrm{v}_{\mathrm{r}} \in \mathrm{V}, \mathrm{r}=1,2,3, \ldots \mathrm{m}, \mathrm{n}$

4. $\left\langle\mu_{\mathrm{rs}}, \mathrm{v}_{\mathrm{rs}}\right\rangle$ denote the degrees of membership and non-membership of edges of $\mathrm{G}$, and is given by 


$$
\left\langle\mu_{r s}, v_{r s}\right\rangle= \begin{cases}\left\langle\min \left(\mu_{i}, \mu_{j}, \mu_{p q}\right), \max \left(v_{i}, v_{j}, v_{p q}\right)\right\rangle & \text { if } i \neq j,\left(v_{i}, v_{j}\right) \notin E_{1} \text { and }\left(u_{p}, u_{q}\right) \in E_{2} \\ \left\langle\min \left(\mu_{p}, \mu_{q}, \mu_{i j}\right), \max \left(v_{p}, v_{q}, v_{i j}\right)\right\rangle & \text { if } p \neq q,\left(u_{p}, u_{q}\right) \notin E_{2} \text { and }\left(v_{i}, v_{j}\right) \in E_{1} \\ \left\langle\min \left(\mu_{i j}, \mu_{p q}\right), \max \left(v_{i j}, v_{p q}\right)\right\rangle & \text { if } i \neq j, p \neq q,\left(v_{i}, v_{j}\right) \in E_{1} \text { and }\left(u_{p}, u_{q}\right) \in E_{2} \\ \langle 0,0\rangle & \text { otherwise }\end{cases}
$$

Definition 2.10. [19] The $\gamma$-product of two intuitionistic fuzzy graphs $G_{1}=\left(V_{1}, E_{1}\right)$ and

$\mathrm{G}_{2}=\left(\mathrm{V}_{2}, \mathrm{E}_{2}\right)$ denoted by $\mathrm{G}_{1} \bullet \mathrm{G}_{2}$ is an intuitionistic fuzzy graphs

$\mathrm{G}=\left(\mathrm{V}, \mathrm{E},\left\langle\mu_{\mathrm{r}}, \mathrm{v}_{\mathrm{r}}\right\rangle,\left\langle\mu_{\mathrm{rs}}, \mathrm{v}_{\mathrm{rs}}\right\rangle\right)$ where

$1 . V=v_{i} u_{p}$ for all $v_{i} \in V_{1}$ and $u_{p} \in V_{2}, V_{1} \cap V_{2}=\emptyset, i=1,2,3, \ldots m, p=1,2,3, \ldots . n$

2.E $=\left\langle v_{i} u_{p}, v_{j} u_{q}\right\rangle$ such that either $\left(v_{i}, v_{j}\right) \in E_{1}$ or $\left(u_{p}, u_{q}\right) \in E_{2}$

3. $\left\langle\mu_{r}, v_{r}\right\rangle$ denote the degrees of membership and non-membership of vertices of $\mathrm{G}$,and is given by $\left\langle\mu_{\mathrm{r}}, \mathrm{v}_{\mathrm{r}}\right\rangle=\left\langle\min \left(\mu_{\mathrm{i}}, \mu_{\mathrm{p}}\right), \max \left(\mathrm{v}_{\mathrm{i}}, \mathrm{v}_{\mathrm{p}}\right)\right\rangle$ for all

$\mathrm{v}_{\mathrm{r}} \in \mathrm{V}, \mathrm{r}=1,2,3, \ldots \mathrm{m}, \mathrm{n}$

4. $\left\langle\mu_{\mathrm{rs}}, \mathrm{v}_{\mathrm{rs}}\right\rangle$ denote the degrees of membership and non-membership of edges of $\mathrm{G}$, and is given by

$$
\left\langle\mu_{\mathrm{rs}}, \mathrm{v}_{\mathrm{rs}}\right\rangle= \begin{cases}\left\langle\min \left(\mu_{\mathrm{i}}, \mu_{\mathrm{j}}, \mu_{\mathrm{pq}}\right), \min \left(\mathrm{v}_{\mathrm{i}}, \mathrm{v}_{\mathrm{j}}, \mathrm{v}_{\mathrm{pq}}\right)\right\rangle & \text { if }\left(\mathrm{v}_{\mathrm{i}}, \mathrm{v}_{\mathrm{j}}\right) \notin \mathrm{E}_{1} \text { and }\left(\mathrm{u}_{\mathrm{p}}, \mathrm{u}_{\mathrm{q}}\right) \in \mathrm{E}_{2} \\ \left\langle\min \left(\mu_{\mathrm{p}}, \mu_{\mathrm{q}}, \mu_{\mathrm{ij}}\right), \min \left(\mathrm{v}_{\mathrm{p}}, \mathrm{v}_{\mathrm{q}}, \mathrm{v}_{\mathrm{ij}}\right)\right\rangle & \text { if }\left(\mathrm{u}_{\mathrm{p}}, \mathrm{u}_{\mathrm{q}}\right) \notin \mathrm{E}_{2} \text { and }\left(\mathrm{v}_{\mathrm{i}}, \mathrm{v}_{\mathrm{j}}\right) \in \mathrm{E}_{1} \\ \left\langle\min \left(\mu_{\mathrm{ij}}, \mu_{\mathrm{pq}}\right), \max \left(\mathrm{v}_{\mathrm{ij}}, \mathrm{v}_{\mathrm{pq}}\right)\right\rangle & \text { if }\left(\mathrm{v}_{\mathrm{i}}, \mathrm{v}_{\mathrm{j}}\right) \in \mathrm{E}_{1} \text { and }\left(\mathrm{u}_{\mathrm{p}}, \mathrm{u}_{\mathrm{q}}\right) \in \mathrm{E}_{2} \\ \langle 0,0\rangle & \text { otherwise }\end{cases}
$$

Definition 2.11. [13] A single valued neutrosophic graph with underlying set $V$ is defined to be a pair $G=(A, B)$ where,

(i) The functions $T_{A} V \rightarrow[0,1], I_{A}: V \rightarrow[0,1]$ and $F_{A}: V \rightarrow[0,1]$ denote the degree of truth-membership, degree of indeterminacy membership, and degree of falsity-membership of the element $v_{i} \in V$, respectively and

$0 \leq \mathrm{T}_{\mathrm{A}}\left(\mathrm{v}_{\mathrm{i}}\right)+\mathrm{I}_{\mathrm{A}}\left(\mathrm{v}_{\mathrm{i}}\right)+\mathrm{F}_{\mathrm{A}}\left(\mathrm{v}_{\mathrm{i}}\right) \leq 3$ for all $\mathrm{v}_{\mathrm{i}} \in \mathrm{V}(\mathrm{i}=1,2, . . \mathrm{n})$

(ii)The functions $\mathrm{T}_{\mathrm{B}}: \mathrm{E} \subseteq \mathrm{V} \times \mathrm{V} \rightarrow[0,1], \mathrm{I}_{\mathrm{B}}: \mathrm{E} \subseteq \mathrm{V} \times \mathrm{V} \rightarrow[0,1]$ and $\mathrm{F}_{\mathrm{B}}: \mathrm{E} \subseteq \mathrm{V} \times \mathrm{V} \rightarrow[0,1]$ are defined by

$$
\begin{aligned}
& \mathrm{T}_{\mathrm{B}}\left(\left\{\mathrm{v}_{\mathrm{i}}, \mathrm{v}_{\mathrm{j}}\right\}\right) \leq \min \left(\mathrm{T}_{\mathrm{A}}\left(\mathrm{v}_{\mathrm{i}}\right), \mathrm{T}_{\mathrm{A}}\left(\mathrm{v}_{\mathrm{j}}\right)\right), \\
& \mathrm{I}_{\mathrm{B}}\left(\left\{\mathrm{v}_{\mathrm{i}}, \mathrm{v}_{\mathrm{j}}\right\}\right) \geq \max \left(\mathrm{I}_{\mathrm{A}}\left(\mathrm{v}_{\mathrm{i}}\right), \mathrm{I}_{\mathrm{A}}\left(\mathrm{v}_{\mathrm{j}}\right)\right) \text { and } \\
& \mathrm{F}_{\mathrm{B}}\left(\left\{\mathrm{v}_{\mathrm{i}}, \mathrm{v}_{\mathrm{j}}\right\}\right) \geq \max \left(\mathrm{F}_{\mathrm{A}}\left(\mathrm{v}_{\mathrm{i}}\right), \mathrm{F}_{\mathrm{A}}\left(\mathrm{v}_{\mathrm{j}}\right)\right)
\end{aligned}
$$

denotes the degree of truth-membership,degree of indeterminacy-membership and degree of falsity-membership of the edge $\left(v_{i}, v_{j}\right) \in$ E respectively, where

$$
0 \leq T_{B}\left(\left\{v_{i}, v_{j}\right\}\right)+I_{B}\left(\left\{v_{i}, v_{j}\right\}\right)+F_{B}\left(\left\{v_{i}, v_{j}\right\}\right) \leq 3 \text { for all }\left\{v_{i}, v_{j}\right\} \in E(i, j=1,2, \ldots n)
$$

Definition 2.12. [13]Let $\mathrm{G}$ be the neutrosophic graph.Let $\mathrm{x}, \mathrm{y} \in \mathrm{V}$. $\mathrm{x}$ dominates $\mathrm{y}$ in $\mathrm{G}$ if

$$
\mu_{1}(x, y)=\min \{\mu(x), \mu(y)\}, \gamma_{1}(x, y)=\min \{\gamma(x), \gamma(y)\} \text { and } \sigma_{1}(x, y)=\min \{\sigma(x), \sigma(y)\}
$$


A subset $D^{N}$ of $V$ is called a dominating set in $G$ if for every vertex $v \notin D^{n}$, there exists $u \in D^{N}$ such that $u$ dominates $\mathrm{v}$.

Definition 2.13. [21] A dominating set $\mathrm{D}^{\mathrm{N}}$ of neutrosophic graph is said to be minimal dominating set if no paper subset of $\mathrm{D}^{\mathrm{N}}$ is a dominating set.

Definition 2.14. [21] Minimum cardinality of a dominating set in a neutrosophic graph $\mathrm{G}$ is called the domination number of $\mathrm{G}$ and is denoted by $\gamma^{\mathrm{N}}(\mathrm{G})($ or $) \gamma^{\mathrm{N}}$.

\section{Dominating energy in neutrosophic graphs}

Dominating energy of a neutrosophic graph using various operations and some theorems on these operations are established here. Let $G=\left(V, E, \mu, \gamma, \sigma, \mu_{1}, \gamma_{1}, \sigma_{1}\right)$ be a dominating neutrosophic graph. Define a dominating neutrosophic adjacency matrix $D^{N}(G)=\left[d_{i j}\right]$, where

$$
d_{i j}= \begin{cases}\left(\mu_{i j}, \gamma_{i j}, \sigma_{i j}\right) & \text { if }\left(v_{i}, v_{j}\right) \in E \\ (1,1) & \text { if } i=j \text { and } v_{i} \in D^{N} \\ (0,0) & \text { otherwise }\end{cases}
$$

This dominating neutrosophic adjacency matrix $D^{N}(G)$ can be written as $D^{N}(G)=\left(\mu_{D^{N}}(G), \gamma_{D^{N}}(G), \sigma_{D^{N}}(G)\right)$, where

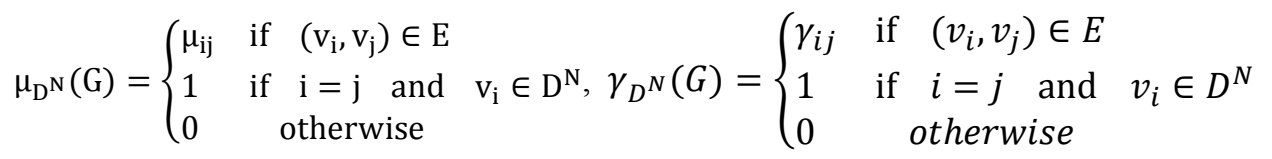

and

$$
\sigma_{D^{N}}(G)= \begin{cases}\sigma_{i j} & \text { if }\left(v_{i}, v_{j}\right) \in E \\ 1 & \text { if } i=j \text { and } \quad v_{i} \in D^{N} \\ 0 & \text { otherwise }\end{cases}
$$

For example, consider the neutrosophic graph $G=(V, E)$, where $V=\left\{v_{1}, v_{2}, v_{3}, v_{4}\right\}, E=$ $\left\{\left(\mathrm{v}_{1} \mathrm{v}_{2}\right),\left(\mathrm{v}_{2} \mathrm{v}_{3}\right),\left(\mathrm{v}_{3} \mathrm{v}_{4}\right),\left(\mathrm{v}_{4} \mathrm{v}_{1}\right)\right\}$ as in Fig. 1

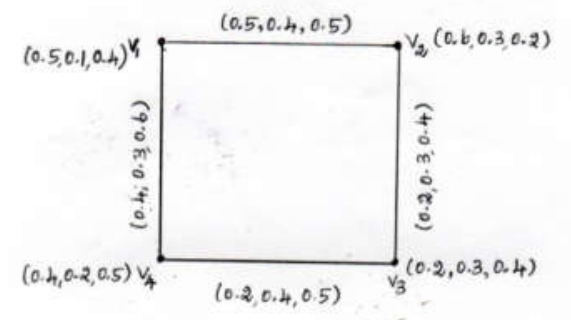

Fig.1

Then, the above dominating neutrosophic graph can be written as $G=\left(V, E, \mu, \gamma, \sigma, \mu_{1}, \gamma_{1}, \sigma_{1}\right)$, where $\mathrm{V}=\left\{\mathrm{v}_{1}, \mathrm{v}_{2}, \mathrm{v}_{3}, \mathrm{v}_{4}\right\}$ and $\mu_{1}, \gamma_{1}, \sigma_{1}$ are given by $\mu_{1}: \mathrm{V} \rightarrow[0,1], \gamma_{1}: \mathrm{V} \rightarrow[0,1]$ and $\sigma_{1}: \mathrm{V} \rightarrow[0,1]$, where

$$
\mu_{1}\left(v_{1}\right)=\min \left[\mu\left(v_{1}, v_{2}\right), \mu\left(v_{1}, v_{4}\right)\right]=\min [0.5,0.4]=0.4
$$




$$
\begin{aligned}
& \mu_{1}\left(v_{2}\right)=\min \left[\mu\left(v_{2}, v_{1}\right), \mu\left(v_{2}, v_{3}\right)\right]=\min [0.5,0.2]=0.2 \\
& \mu_{1}\left(v_{3}\right)=\min \left[\mu\left(v_{3}, v_{2}\right), \mu\left(v_{3}, v_{4}\right)\right]=\min [0.2,0.2]=0.2 \\
& \mu_{1}\left(v_{4}\right)=\min \left[\mu\left(v_{4}, v_{1}\right), \mu\left(v_{4}, v_{3}\right)\right]=\min [0.4,0.2]=0.2 \\
& \gamma_{1}\left(v_{1}\right)=\max \left[\gamma\left(v_{1}, v_{2}\right), \gamma\left(v_{1}, v_{4}\right)\right]=\max [0.4,0.3]=0.4 \\
& \gamma_{1}\left(v_{2}\right)=\max \left[\gamma\left(v_{2}, v_{1}\right), \gamma\left(v_{2}, v_{3}\right)\right]=\max [0.4,0.3]=0.4 \\
& \gamma_{1}\left(v_{3}\right)=\max \left[\gamma\left(v_{3}, v_{2}\right), \gamma\left(v_{3}, v_{4}\right)\right]=\max [0.3,0.4]=0.4 \\
& \gamma_{1}\left(v_{4}\right)=\max \left[\gamma\left(v_{4}, v_{1}\right), \gamma\left(v_{4}, v_{3}\right)\right]=\max [0.3,0.4]=0.4 \\
& \sigma_{1}\left(v_{1}\right)=\max \left[\sigma\left(v_{1}, v_{2}\right), \sigma\left(v_{1}, v_{4}\right)\right]=\max [0.5,0.6]=0.6 \\
& \sigma_{1}\left(v_{2}\right)=\max \left[\sigma\left(v_{2}, v_{1}\right), \sigma\left(v_{2}, v_{3}\right)\right]=\max [0.5,0.4]=0.6 \\
& \sigma_{1}\left(v_{3}\right)=\max \left[\sigma\left(v_{3}, v_{2}\right), \sigma\left(v_{3}, v_{4}\right)\right]=\max [0.4,0.5]=0.5 \\
& \sigma_{1}\left(v_{4}\right)=\max \left[\sigma\left(v_{4}, v_{1}\right), \sigma\left(v_{4}, v_{3}\right)\right]=\max [0.6,0.5]=0.6
\end{aligned}
$$

Here, $v_{2}$ dominates $v_{3}$ because

$$
\begin{gathered}
\mu\left(\mathrm{v}_{2} \mathrm{v}_{3}\right) \leq \mu_{1}\left(\mathrm{v}_{2}\right) \wedge \mu_{1}\left(\mathrm{v}_{3}\right) 0.2 \leq 0.2 \wedge 0.29 \\
\gamma\left(\mathrm{v}_{2} \mathrm{v}_{3}\right) \leq \gamma\left(\mathrm{v}_{2}\right) \wedge \gamma\left(\mathrm{v}_{3}\right) 0.3 \leq 0.4 \wedge 0.4 \\
\sigma\left(\mathrm{v}_{2} \mathrm{v}_{3}\right) \leq \sigma\left(\mathrm{v}_{2}\right) \wedge \sigma\left(\mathrm{v}_{3}\right) 0.4 \leq 0.5 \wedge 0.5
\end{gathered}
$$

Here, $\mathrm{v}_{3}$ dominates $\mathrm{v}_{4}$ because

$$
\begin{aligned}
& \mu\left(\mathrm{v}_{3} \mathrm{v}_{4}\right) \leq \mu_{1}\left(\mathrm{v}_{3}\right) \wedge \mu_{1}\left(\mathrm{v}_{4}\right) 0.2 \leq 0.2 \wedge 0.2 \\
& \gamma\left(\mathrm{v}_{3} \mathrm{v}_{4}\right) \leq \gamma_{1}\left(\mathrm{v}_{3}\right) \wedge \gamma_{1}\left(\mathrm{v}_{4}\right) 0.4 \leq 0.4 \wedge 0.4 \\
& \sigma\left(\mathrm{v}_{3} \mathrm{v}_{4}\right) \leq \sigma\left(\mathrm{v}_{3}\right) \wedge \sigma\left(\mathrm{v}_{4}\right) 0.5 \leq 0.5 \wedge 0.6 \\
& V=\left\{\mathrm{v}_{1}, \mathrm{v}_{2}, \mathrm{v}_{3}, \mathrm{v}_{4}\right\}, \mathrm{D}^{\mathrm{N}}=\left\{\mathrm{v}_{2}, \mathrm{v}_{3}\right\} \text { and } \mathrm{V}-\mathrm{D}^{\mathrm{N}}=\left\{\mathrm{v}_{1}, \mathrm{v}_{4}\right\}
\end{aligned}
$$

$\left|D^{N}\right|=2=$ sum of dominating elements

$$
D^{N}(G)=\left[\begin{array}{llll}
(0,0,0) & (0.5,0.4,0.5) & (0,0,0) & (0.4,0.2,0.6) \\
(0.5,0.4,0.5) & (1,1,1) & (0.2,0.3,0.4) & (0,0,0) \\
(0,0,0) & (0.2,0.3,0.4) & (1,1,1) & (0.4,0.3) \\
(0.2,0.3) & (0.3,0.1) & (0.2,0.4,0.5) & (0,0,0)
\end{array}\right] \text { where }
$$

$\mu_{\mathrm{D}^{\mathrm{N}}}(\mathrm{G})=\left[\begin{array}{llll}1 & 0.5 & 0 & 0.4 \\ 0.5 & 1 & 0.2 & 0 \\ 0 & 0.2 & 1 & 0.2 \\ 0.4 & 0 & 0.2 & 0\end{array}\right] \quad \gamma_{\mathrm{D}^{\mathrm{N}}}(\mathrm{G})=\left[\begin{array}{llll}0 & 0.4 & 0 & 0.2 \\ 0.4 & 1 & 0.3 & 0 \\ 0 & 0.3 & 1 & 0.4 \\ 0.3 & 0 & 0.4 & 0\end{array}\right]$ and $\sigma_{\mathrm{D}^{\mathrm{N}}}(\mathrm{G})=\left[\begin{array}{llll}0 & 0.5 & 0 & 0.6 \\ 0.5 & 1 & 0.4 & 0 \\ 0 & 0.4 & 1 & 0.5 \\ 0.6 & 0 & 0.5 & 0\end{array}\right]$

3.1 Dominating energy in operations on neutrosophic graph Dominating energy in complement of neutrosophic graph: 
The complement of neutrosophic graph $G=(V, E)$ is neutrosophic graph, $\bar{G}=\langle\bar{V}, \bar{E}\rangle$, where $\bar{\mu}_{1 \mathrm{i}}=$ $\mu_{1 \mathrm{i}}, \bar{\gamma}_{1 \mathrm{i}}=\gamma_{1 \mathrm{i}}$ and $\bar{\sigma}_{1 \mathrm{i}}=\sigma_{\mathrm{ij}}$, for all $\mathrm{i}=1,2, \ldots . . \mathrm{n} \bar{\mu}_{2 \mathrm{ij}}=\mu_{1 \mathrm{i}} \mu_{1 \mathrm{j}}-\mu_{\mathrm{ij}}, \bar{\gamma}_{2 \mathrm{ij}}=\gamma_{1 \mathrm{i}} \gamma_{1 \mathrm{j}}-\gamma_{2 \mathrm{ij}}$ and $\bar{\sigma}_{2 \mathrm{ij}}=\sigma_{1 \mathrm{i}} \sigma_{1 \mathrm{j}}-\sigma_{2 \mathrm{ij}}$, for all $\mathrm{i}, \mathrm{j}=1,2, \ldots . \mathrm{n}$

First we find the dominating energy of neutrosophic graph $G(V, E)$.

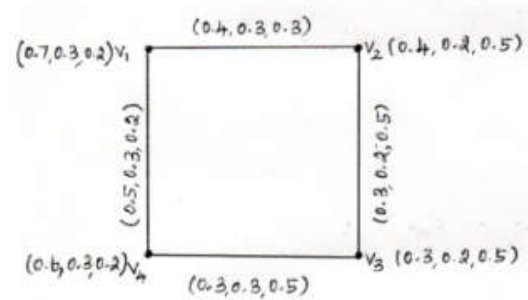

Figure $2: \mathrm{G}=(\mathrm{V}, \mathrm{E})$

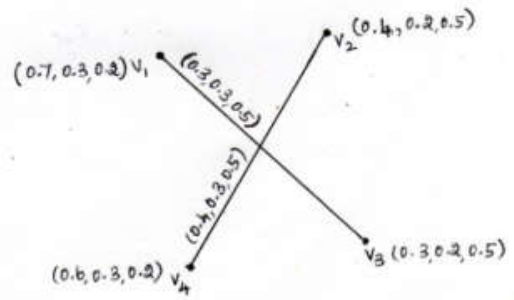

Figure $3: \overline{\mathrm{G}}=\langle\overline{\mathrm{V}}, \overline{\mathrm{E}}\rangle$

Consider a dominating neutrosophic graph $G=\left(V, E, \mu, \gamma, \sigma, \mu_{1}, \gamma_{1}, \sigma_{1}\right)$, where $V=\left\{v_{1}, v_{2}, v_{3}, v_{4}\right\}$ and $\mu_{1}, \gamma_{1}, \sigma_{1}$ are given by $\mu_{1}: V \rightarrow[0,1], \gamma_{1}: V \rightarrow[0,1]$ and $\sigma_{1}: V \rightarrow[0,1]$ where

$$
\begin{aligned}
& \mu_{1}\left(v_{1}\right)=\min \left[\mu\left(v_{1} v_{2}\right), \mu\left(v_{1} v_{4}\right)\right]=\min [0.4,0.5]=0.4 \\
& \mu_{1}\left(v_{2}\right)=\min \left[\mu\left(v_{2} v_{1}\right), \mu\left(v_{2} v_{3}\right)\right]=\min [0.4,0.3]=0.3 \\
& \mu_{1}\left(v_{3}\right)=\min \left[\mu\left(v_{3} v_{2}\right), \mu\left(v_{3} v_{4}\right)\right]=\min [0.3,0.2]=0.2 \\
& \mu_{1}\left(v_{4}\right)=\min \left[\mu\left(v_{4} v_{1}\right), \mu\left(v_{4} v_{3}\right)\right]=\min [0.2,0.5]=0.2 \\
& \gamma_{1}\left(v_{1}\right)=\max \left[\gamma\left(v_{1} v_{2}\right), \gamma\left(v_{1} v_{4}\right)\right]=\max [0.3,0.3]=0.3 \\
& \gamma_{1}\left(v_{2}\right)=\max \left[\gamma\left(v_{2} v_{1}\right), \gamma\left(v_{2} v_{3}\right)\right]=\max [0.3,0.2]=0.3 \\
& \gamma_{1}\left(v_{3}\right)=\max \left[\gamma\left(v_{3} v_{2}\right), \gamma\left(v_{3} v_{4}\right)\right]=\max [0.2,0.3]=0.3 \\
& \gamma_{1}\left(v_{4}\right)=\max \left[\gamma\left(v_{4} v_{1}\right), \gamma\left(v_{4} v_{3}\right)\right]=\max [0.3,0.3]=0.3 \\
& \sigma_{1}\left(v_{1}\right)=\max \left[\sigma\left(v_{1} v_{2}\right), \sigma\left(v_{1} v_{4}\right)\right]=\max [0.3,0.2]=0.3 \\
& \sigma_{1}\left(v_{2}\right)=\max \left[\sigma\left(v_{2} v_{1}\right), \sigma\left(v_{2} v_{3}\right)\right]=\max [0.5,0.5]=0.5 \\
& \sigma_{1}\left(v_{3}\right)=\max \left[\sigma\left(v_{3} v_{2}\right), \sigma\left(v_{3} v_{4}\right)\right]=\max [0.5,0.5]=0.5 \\
& \sigma_{1}\left(v_{4}\right)=\max \left[\sigma\left(v_{4} v_{1}\right), \sigma\left(v_{4} v_{3}\right)\right]=\max [0.5,0.2]=0.5
\end{aligned}
$$

Here, $v_{3}$ dominates $v_{4}$ because

$$
\begin{gathered}
\mu\left(\mathrm{v}_{3} \mathrm{v}_{4}\right) \leq \mu_{1}\left(\mathrm{v}_{3}\right) \wedge \mu_{1}\left(\mathrm{v}_{4}\right) 0.2 \leq 0.2 \wedge 0.2 \\
\gamma\left(\mathrm{v}_{3} \mathrm{v}_{4}\right) \leq \gamma\left(\mathrm{v}_{3}\right) \wedge \gamma\left(\mathrm{v}_{4}\right) 0.3 \leq 0.3 \wedge 0.3 \\
\sigma\left(\mathrm{v}_{3} \mathrm{v}_{4}\right) \leq \sigma\left(\mathrm{v}_{3}\right) \wedge \sigma\left(\mathrm{v}_{4}\right) 0.5 \leq 0.5 \wedge 0.5 \\
\mathrm{~V}=\left\{\mathrm{v}_{1}, \mathrm{v}_{2}, \mathrm{v}_{3}, \mathrm{v}_{4}\right\}, \mathrm{D}^{\mathrm{N}}=\left\{\mathrm{v}_{3}\right\} \text { and } \mathrm{V}-\mathrm{D}^{\mathrm{N}}=\left\{\mathrm{v}_{1}, \mathrm{v}_{2}, \mathrm{v}_{4}\right\}
\end{gathered}
$$


$\left|D^{\mathrm{N}}\right|=1=$ sum of dominating elements

$$
\begin{aligned}
& \mathrm{D}^{\mathrm{N}}(\mathrm{G})=\left[\begin{array}{llll}
(0,0,0) & (0.4,0.3,0.3) & (0,0,0) & (0.2,0.3,0.5) \\
(0.4,0.3,0.3) & (0,0,0) & (0.3,0.2,0.5) & (0,0,0) \\
(0,0,0) & (0.3,0.2,0.5) & (1,1,1) & (0.2,0.3,0.5) \\
(0.5,0.3,0.2) & (0,0,0) & (0.2,0.3,0.5) & (0,0,0)
\end{array}\right], \text { where } \\
& {\left[\begin{array}{lllll}
0 & 0.4 & 0 & 0.2 \\
0.4 & 0 & 0.3 & 0 \\
0 & 0.3 & 1 & 0.2 \\
0.5 & 0 & 0.2 & 0
\end{array}\right], \gamma_{\mathrm{D}^{\mathrm{N}}}(\mathrm{G})=\left[\begin{array}{llll}
0 & 0.3 & 0 & 0.3 \\
0.3 & 0 & 0.2 & 0 \\
0 & 0.2 & 1 & 0.3 \\
0.3 & 0 & 0.3 & 0
\end{array}\right] \text { and } \sigma_{\mathrm{D}^{\mathrm{N}}}(\mathrm{G})=\left[\begin{array}{llll}
0 & 0.3 & 0 & 0.5 \\
0.3 & 0 & 0.5 & 0 \\
0 & 0.5 & 1 & 0.5 \\
0.2 & 0 & 0.5 & 0
\end{array}\right] }
\end{aligned}
$$

Eigen values of $\mu_{D^{N}}(G)=\{-0.5549,0.4064,1.1431,0,0054\}=$ spectrum of $\mu_{D^{N}}(G)$

Eigen values of $\gamma_{D^{N}}(G)=\{-0.4692,0.3414,1.1327,-0.0049\}=$ spectrum of $\gamma_{D^{N}}(G)$

Eigen values of $\sigma_{D^{N}}(G)=\{1.3981,-0.6218,0.1940,0.0296\}=$ spectrum of $\sigma_{D^{N}}(G)$

Dominating energy of neutrosophic graph

$$
\mathrm{G}=(\mathrm{V}, \mathrm{E})=\left[\sum_{\lambda_{\mathrm{i}} \in \mathrm{X}}\left|\lambda_{\mathrm{i}}\right|, \sum_{\delta_{\mathrm{i}} \in \mathrm{Y}}\left|\delta_{\mathrm{i}}\right|, \sum_{\rho_{\mathrm{i}} \in \mathrm{Z}}\left|\rho_{\mathrm{i}}\right|\right]=[2.1098,1.9482,2.2435]
$$

Now we find the dominating energy of neutrosophic graph $G(V, E)$.

Consider a dominating neutrosophic graph $G=\left(V, E, \mu, \gamma, \sigma, \mu_{1}, \gamma_{1}, \sigma_{1}\right)$ where $V=\left\{v_{1}, v_{2}, v_{3}, v_{4}\right\}$ and $\mu_{1}, \gamma_{1}, \sigma_{1}$ are given by $\mu_{1}: \mathrm{V} \rightarrow[0,1], \gamma_{1}: \mathrm{V} \rightarrow[0,1]$ and $\sigma_{1}: \mathrm{V} \rightarrow[0,1]$ where

$$
\begin{aligned}
& \mu_{1}\left(v_{1}\right)=\min \left[\mu\left(v_{1} v_{3}\right)\right]=\min [0.3]=0.3 \\
& \mu_{1}\left(v_{2}\right)=\min \left[\mu\left(v_{2} v_{4}\right)\right]=\min [0.4]=0.4 \\
& \mu_{1}\left(v_{3}\right)=\min \left[\mu\left(v_{3} v_{1}\right)\right]=\min [0.3]=0.3 \\
& \mu_{1}\left(v_{4}\right)=\min \left[\mu\left(v_{4} v_{2}\right]=\min [0.4]=0.4\right. \\
& \gamma_{1}\left(v_{1}\right)=\max \left[\gamma\left(v_{1} v_{3}\right)\right]=\max [0.3]=0.3 \\
& \gamma_{1}\left(v_{2}\right)=\max \left[\gamma\left(v_{2} v_{4}\right)\right]=\max [0.3]=0.3 \\
& \gamma_{1}\left(v_{3}\right)=\max \left[\gamma\left(v_{3} v_{1}\right)\right]=\max [0.3]=0.3 \\
& \gamma_{1}\left(v_{4}\right)=\max \left[\gamma\left(v_{4} v_{2}\right)\right]=\max [0.3]=0.3 \\
& \sigma_{1}\left(v_{1}\right)=\max \left[\sigma\left(v_{1} v_{3}\right)\right]=\max [0.5]=0.5 \\
& \sigma_{1}\left(v_{2}\right)=\max \left[\sigma\left(v_{2} v_{4}\right)\right]=\max [0.5]=0.5 \\
& \sigma_{1}\left(v_{3}\right)=\max \left[\sigma\left(v_{3} v_{1}\right)\right]=\max [0.5]=0.5 \\
& \sigma_{1}\left(v_{4}\right)=\max \left[\sigma\left(v_{4} v_{2}\right)\right]=\max [0.5]=0.5
\end{aligned}
$$

Here, $\mathrm{v}_{1}$ dominates $\mathrm{v}_{3}$ because 


$$
\begin{gathered}
\mu\left(\mathrm{v}_{1} \mathrm{v}_{3}\right) \leq \mu_{1}\left(\mathrm{v}_{1}\right) \wedge \mu_{1}\left(\mathrm{v}_{3}\right) 0.3 \leq 0.3 \wedge 0.3 \\
\gamma\left(\mathrm{v}_{1} \mathrm{v}_{3}\right) \leq \gamma\left(\mathrm{v}_{1}\right) \wedge \gamma\left(\mathrm{v}_{3}\right) 0.3 \leq 0.3 \wedge 0.3 \\
\sigma\left(\mathrm{v}_{1} \mathrm{v}_{3}\right) \leq \sigma\left(\mathrm{v}_{1}\right) \wedge \sigma\left(\mathrm{v}_{3}\right) 0.5 \leq 0.5 \wedge 0.5
\end{gathered}
$$

Here, $\mathrm{v}_{2}$ dominates $\mathrm{v}_{4}$ because

$$
\begin{gathered}
\mu\left(\mathrm{v}_{2} \mathrm{v}_{4}\right) \leq \mu_{1}\left(\mathrm{v}_{2}\right) \wedge \mu_{1}\left(\mathrm{v}_{4}\right) 0.4 \leq 0.4 \wedge 0.4 \\
\gamma\left(\mathrm{v}_{2} \mathrm{v}_{4}\right) \leq \gamma\left(\mathrm{v}_{2}\right) \wedge \gamma\left(\mathrm{v}_{4}\right) 0.3 \leq 0.3 \wedge 0.3 \\
\sigma\left(\mathrm{v}_{2} \mathrm{v}_{4}\right) \leq \sigma\left(\mathrm{v}_{2}\right) \wedge \sigma\left(\mathrm{v}_{4}\right) 0.5 \leq 0.5 \wedge 0.5
\end{gathered}
$$

$\mathrm{V}=\left\{\mathrm{v}_{1}, \mathrm{v}_{2}, \mathrm{v}_{3}, \mathrm{v}_{4}\right\}, \mathrm{D}^{\mathrm{N}}=\left\{\mathrm{v}_{1}, \mathrm{v}_{2}\right\}$ and $\mathrm{V}-\mathrm{D}^{\mathrm{N}}=\left\{\mathrm{v}_{3}, \mathrm{v}_{4}\right\}$

$\left|D^{\mathrm{N}}\right|=2=$ sum of dominating elements

$$
\begin{aligned}
& D^{N}(G)=\left[\begin{array}{llll}
(1,1,1) & (0,0,0) & (0.3,0.3,0.5) & (0,0,0) \\
(0,0,0) & (1,1,1) & (0,0,0) & (0.4,0.3,0.5) \\
(0.3,0.3,0.5) & (0,0,0) & (0,0,0) & (0,0,0) \\
(0,0,0) & (0.4,0.3,0.5) & (0,0,0) & (0,0,0)
\end{array}\right] \text { where } \\
& \mu_{D^{N}}(G)=\left[\begin{array}{llll}
1 & 0 & 0.3 & 0 \\
0 & 1 & 0 & 0.4 \\
0.3 & 0 & 0 & 0 \\
0 & 0.4 & 0 & 0
\end{array}\right], \gamma_{D^{N}}(G)=\left[\begin{array}{llll}
1 & 0 & 0.3 & 0 \\
0 & 1 & 0 & 0.3 \\
0.3 & 0 & 0 & 0 \\
0 & 0.3 & 0 & 0
\end{array}\right] \text { and } \sigma_{D^{N}}(G)=\left[\begin{array}{llll}
1 & 0 & 0.5 & 0 \\
0 & 1 & 0 & 0.5 \\
0.5 & 1 & 0 & 0 \\
0 & 0.5 & 0 & 0
\end{array}\right]
\end{aligned}
$$

Eigen values of $\mu_{D^{N}}(G)=\{1.0830,-0.0830,1.4031,-0.1403\}=$ spectrum of $\mu_{D^{N}}(G)$

Eigen values of $\gamma_{D^{N}}(G)=\{1.0830,-0.0830,1.0830,-0.0830\}=$ spectrum of $\gamma_{D^{N}}(G)$

Eigen values of $\sigma_{D^{N}}(G)=\{1.2071,-0.2071,1.2071,-0.2071\}=$ spectrum of $\sigma_{D^{N}}(G)$

Dominating energy of complement of neutrosophic graph

$$
\mathrm{G}=(\mathrm{V}, \mathrm{E})=\left[\sum_{\lambda_{\mathrm{i}} \in \mathrm{X}}\left|\lambda_{\mathrm{i}}\right|, \sum_{\delta_{\mathrm{i}} \in \mathrm{Y}}\left|\delta_{\mathrm{i}}\right|, \sum_{\rho_{\mathrm{i}} \in \mathrm{Z}}\left|\rho_{\mathrm{i}}\right|\right]=[2.7094,2.332,2.8284]
$$

\subsection{Dominating energy in union of neutrosophic graph}

Let $G_{1}=\left\langle V_{1}, E_{1}\right\rangle$ and $G_{2}=\left\langle V_{2}, E_{2}\right\rangle$ be two neutrosophic Graphs with $V_{1} \cap V_{2}=\emptyset$ and $G=G_{1} \cup G_{2}=$ $\left\langle V_{1} \cup V_{2}, E_{1} \cup E_{2}\right\rangle$ be the union of $G_{1}$ and $G_{2}$. Then the union of neutrosophic graphs $G_{1}$ and $G_{2}$ is neutrosophic graph defined by

$$
\begin{aligned}
& \left(\mu_{1} \cup \mu_{1 \prime}^{\prime}\right)(v)=\left\{\begin{array}{lll}
\mu_{1}(v) & \text { if } & v \in v_{1}-v_{2} \\
\mu_{1}(v) & \text { if } & v \in v_{2}-v_{1}
\end{array}, \quad\left(\gamma_{1} \cup \gamma_{1 \prime}\right)(v)=\left\{\begin{array}{lll}
\gamma_{1}(v) & \text { if } & v \in v_{1}-v_{2} \\
\gamma_{1 \prime}(v) & \text { if } & v \in v_{2}-v_{1}
\end{array}\right.\right. \\
& \left(\sigma_{1} \cup \sigma_{1^{\prime}}\right)(v)=\left\{\begin{array}{lll}
\sigma_{1}(v) & \text { if } & v \in v_{1}-v_{2} \\
\sigma_{1 \prime}(v) & \text { if } & v \in v_{2}-v_{1}
\end{array} \text { and }\left(\mu_{2} \cup \mu_{2 \prime}\right)\left(v_{i}, v_{j}\right)=\left\{\begin{array}{lll}
\mu_{2}\left(e_{i j}\right) & \text { if } & e_{i j} \in E_{1}-E_{2} \\
\mu_{2 \prime}\left(e_{i j}\right) & \text { if } & e_{i j} \in E_{2}-E_{1}
\end{array}\right.\right.
\end{aligned}
$$

where $\left(\mu_{1}, \gamma_{1}, \sigma_{1}\right)$ and $\left(\mu_{1}, \gamma_{1}, \sigma_{1}\right)$ refer the vertex truth-membership, indeterminacy-membership and falsitymembership of $G_{1}$ and $G_{2}$ respectively, $\left(\mu_{2}, \gamma_{2}, \sigma_{2}\right)$ and $\left(\mu_{2 \prime}, \gamma_{2 \prime}, \sigma_{2 \prime}\right)$ refer the edge truth-membership, indeterminacy-membership and falsity-membership of $G_{1}$ and $G_{2}$ respectively. 
First we find the dominating energy of neutrosophic graph $G_{1}(V, E)$

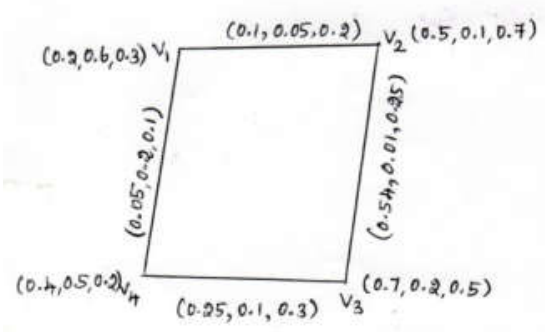

Fig. 4: $G_{1}$

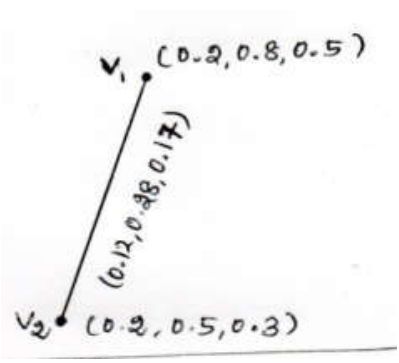

Fig.5: $G_{2}$

Consider a dominating neutrosophic graph $G=\left(V, E, \mu, \gamma, \sigma, \mu_{1}, \gamma_{1}, \sigma_{1}\right)$, where $V=\left\{v_{1}, v_{2}, v_{3}, v_{4}\right\}$ and $\mu_{1}, \gamma_{1}, \sigma_{1}$ are given by $\mu_{1}: \mathrm{V} \rightarrow[0,1], \gamma_{1}: \mathrm{V} \rightarrow[0,1]$ and $\sigma_{1}: \mathrm{V} \rightarrow[0,1]$ where

$$
\begin{gathered}
\mu_{1}\left(v_{1}\right)=\min \left[\mu\left(v_{1} v_{2}\right), \mu\left(v_{1} v_{4}\right)\right]=\min [0.1,0.05]=0.05 \\
\mu_{1}\left(v_{2}\right)=\min \left[\mu\left(v_{2} v_{1}\right), \mu\left(v_{2} v_{3}\right)\right]=\min [0.54,0.1]=0.1 \\
\mu_{1}\left(v_{3}\right)=\min \left[\mu\left(v_{3} v_{2}\right), \mu\left(v_{3} v_{4}\right)\right]=\min [0.54,0.25]=0.25 \\
\mu_{1}\left(v_{4}\right)=\min \left[\mu\left(v_{4} v_{1}\right), \mu\left(v_{4} v_{3}\right)\right]=\min [0.05,0.25]=0.05 \\
\gamma_{1}\left(v_{1}\right)=\max \left[\gamma\left(v_{1} v_{2}\right), \gamma\left(v_{1} v_{4}\right)\right]=\max [0.05,0.2]=0.2 \\
\gamma_{1}\left(v_{2}\right)=\max \left[\gamma\left(v_{2} v_{1}\right), \gamma\left(v_{2} v_{3}\right)\right]=\max [0.05,0.01]=0.05 \\
\gamma_{1}\left(v_{3}\right)=\max \left[\gamma\left(v_{3} v_{2}\right), \gamma\left(v_{3} v_{4}\right)\right]=\max [0.01,0.1]=0.1 \\
\gamma_{1}\left(v_{4}\right)=\max \left[\gamma\left(v_{4} v_{1}\right), \gamma\left(v_{4} v_{3}\right)\right]=\max [0.2,0.1]=0.2 \\
\sigma_{1}\left(v_{1}\right)=\max \left[\sigma\left(v_{1} v_{2}\right), \sigma\left(v_{1} v_{4}\right)\right]=\max [0.2,0.1]=0.2 \\
\sigma_{1}\left(v_{2}\right)=\max \left[\sigma\left(v_{2} v_{1}\right), \sigma\left(v_{2} v_{3}\right)\right]=\max [0.2,0.25]=0.25 \\
\sigma_{1}\left(v_{3}\right)=\max \left[\sigma\left(v_{3} v_{2}\right), \sigma\left(v_{3} v_{4}\right)\right]=\max [0.25,0.3]=0.3 \\
\sigma_{1}\left(v_{4}\right)=\max \left[\sigma\left(v_{4} v_{1}\right), \sigma\left(v_{4} v_{3}\right)\right]=\max [0.1,0.3]=0.3
\end{gathered}
$$

Here, $\mathrm{v}_{1}$ dominates $\mathrm{v}_{4}$ because

$$
\begin{gathered}
\mu\left(\mathrm{v}_{1} \mathrm{v}_{4}\right) \leq \mu_{1}\left(\mathrm{v}_{1}\right) \wedge \mu_{1}\left(\mathrm{v}_{4}\right) 0.05 \leq 0.05 \wedge 0.05 \\
\gamma\left(\mathrm{v}_{1} \mathrm{v}_{4}\right) \leq \gamma\left(\mathrm{v}_{1}\right) \wedge \gamma\left(\mathrm{v}_{4}\right) 0.2 \leq 0.2 \wedge 0.2 \\
\sigma\left(\mathrm{v}_{1} \mathrm{v}_{4}\right) \leq \sigma\left(\mathrm{v}_{1}\right) \wedge \sigma\left(\mathrm{v}_{4}\right) 0.05 \leq 0.2 \wedge 0.3 \\
\mathrm{~V}=\left\{\mathrm{v}_{1}, \mathrm{v}_{2}, \mathrm{v}_{3}, \mathrm{v}_{4}\right\}, \mathrm{D}^{\mathrm{N}}=\left\{\mathrm{v}_{1}\right\} \text { and } \mathrm{V}-\mathrm{D}^{\mathrm{N}}=\left\{\mathrm{v}_{2}, \mathrm{v}_{3}, \mathrm{v}_{4}\right\} \\
\left|\mathrm{D}^{\mathrm{N}}\right|=1=\text { sum of dominating elements }
\end{gathered}
$$




$$
\begin{aligned}
\mathrm{D}^{\mathrm{N}}\left(\mathrm{G}_{1}\right) & =\left[\begin{array}{llll}
(1,1,1) & (0.1,0.05,0.2) & (0,0,0) & (0.05,0.5,0.2) \\
(0.1,0.05,0.2) & (0,0,0) & (0.54,0.01,0.25) & (0,0,0) \\
(0,0,0) & (0.54,0.01,0.25) & (0,0,0) & (0.25,0.1,0.3) \\
(0.05,0.5,0.2) & (0,0,0) & (0.25,0.1,0.3) & (0,0,0)
\end{array}\right] \text { where } \\
\mu_{D^{N}}\left(G_{1}\right) & =\left[\begin{array}{llll}
1 & 0.1 & 0 & 0.05 \\
0.1 & 0 & 0.54 & 0 \\
0 & 0.54 & 0 & 0.25 \\
0.05 & 0 & 0.25 & 0
\end{array}\right] \gamma_{\mathrm{D}^{\mathrm{N}}}\left(\mathrm{G}_{1}\right)=\left[\begin{array}{llll}
1 & 0.05 & 0 & 0.5 \\
0.05 & 0 & 0.01 & 0 \\
0 & 0.01 & 0 & 0.1 \\
0.5 & 0 & 0.1 & 0
\end{array}\right] \text { and } \\
\sigma_{\mathrm{D}^{\mathrm{N}}}\left(\mathrm{G}_{1}\right) & =\left[\begin{array}{llll}
1 & 0.2 & 0 & 0.2 \\
0.2 & 0 & 0.25 & 0 \\
0 & 0.25 & 0 & 0.3 \\
0.2 & 0 & 0.3 & 0
\end{array}\right]
\end{aligned}
$$

Eigen values of $\mu_{D^{N}}\left(G_{1}\right)=\{1.0186,-0.5989,0.5803,0\}=$ spectrum of $\mu_{D^{N}}\left(G_{1}\right)$

Eigen values of $\gamma_{D^{N}}\left(G_{1}\right)=\{1.2101,-0.2442,0.0341,0\}=$ spectrum of $\gamma_{D^{N}}\left(G_{1}\right)$

Eigen values of $\sigma_{D^{N}}\left(G_{1}\right)=\{1.0846,-0.4194,0.3354,-0.0006\}=$ spectrum of $\sigma_{D^{N}}\left(G_{1}\right)$

Dominating energy of neutrosophic graph

$\mathrm{G}=(\mathrm{V}, \mathrm{E})=\left[\sum_{\lambda_{\mathrm{i}} \in \mathrm{X}}\left|\lambda_{\mathrm{i}}\right|, \sum_{\delta_{\mathrm{i}} \in \mathrm{Y}}\left|\delta_{\mathrm{i}}\right|, \sum_{\rho_{\mathrm{i}} \in \mathrm{Z}}\left|\rho_{\mathrm{i}}\right|\right]=[2.1978,1.4884,1.84]$

Also we find the dominating energy of neutrosophic graph $G_{2}(V, E)$ :

Let $\mathrm{V}=\left\{\mathrm{v}_{1}, \mathrm{v}_{2}\right\}$

$$
\begin{aligned}
& \mu_{1}\left(v_{1}\right)=\min \left[\mu\left(v_{1} v_{2}\right]=\max [0.12]=0.12\right. \\
& \mu_{1}\left(v_{2}\right)=\min \left[\mu\left(v_{2} v_{1}\right)\right]=\max [0.12]=0.12 \\
& \gamma_{1}\left(v_{1}\right)=\max \left[\gamma\left(v_{1} v_{2}\right)\right]=\min [0.28]=0.28 \\
& \gamma_{1}\left(v_{2}\right)=\max \left[\gamma\left(v_{2} v_{1}\right)\right]=\min [0.28]=0.28 \\
& \sigma_{1}\left(v_{1}\right)=\max \left[\sigma\left(v_{1} v_{2}\right)\right]=\min [0.17]=0.17 \\
& \sigma_{1}\left(v_{2}\right)=\max \left[\sigma\left(v_{2} v_{1}\right)\right]=\min [0.17]=0.17
\end{aligned}
$$

Here, $\mathrm{v}_{1}$ dominates $\mathrm{v}_{2}$ because

$$
\begin{aligned}
& \mu\left(\mathrm{v}_{1} \mathrm{v}_{2}\right) \leq \mu_{1}\left(\mathrm{v}_{1}\right) \wedge \mu_{1}\left(\mathrm{v}_{2}\right) 0.12 \leq 0.12 \wedge 0.12 \\
& \gamma\left(\mathrm{v}_{1} \mathrm{v}_{2}\right) \leq \gamma_{1}\left(\mathrm{v}_{1}\right) \wedge \gamma_{1}\left(\mathrm{v}_{2}\right) 0.28 \leq 0.28 \wedge 0.28 \\
& \sigma\left(\mathrm{v}_{1} \mathrm{v}_{2}\right) \leq \sigma_{1}\left(\mathrm{v}_{1}\right) \wedge \sigma_{1}\left(\mathrm{v}_{2}\right) 0.17 \leq 0.17 \wedge 0.17
\end{aligned}
$$

Here, $\mathrm{V}=\left\{\mathrm{v}_{1}, \mathrm{v}_{2}\right\}$ and $\mathrm{D}^{\mathrm{N}}=\left\{\mathrm{v}_{1}\right\} ; \mathrm{V}-\mathrm{D}^{\mathrm{N}}=\left\{\mathrm{v}_{2}\right\}$

$\left|D^{\mathrm{N}}\right|=1=$ sum of dominating elements.

$$
D^{N}\left(G_{2}\right)=\left[\begin{array}{ll}
(1,1,1) & (0.12,0.28,0.17) \\
(0.12,0.28,0.17) & (0,0,0)
\end{array}\right], \text { where }
$$


$\mu_{D^{N}}\left(G_{2}\right)=\left[\begin{array}{ll}1 & 0.12 \\ 0.12 & 0\end{array}\right] \gamma_{D^{N}}\left(G_{2}\right)=\left[\begin{array}{ll}1 & 0.28 \\ 0.28 & 0\end{array}\right]$ and $\sigma_{D^{N}}\left(G_{2}\right)=\left[\begin{array}{ll}1 & 0.17 \\ 0.17 & 0\end{array}\right]$

Eigen values of $\mu_{D^{N}}\left(G_{2}\right)=\{1.0142,-0.0141\}=$ spectrum of $\mu_{D^{N}}\left(G_{2}\right)$

Eigen values of $\gamma_{D^{N}}\left(G_{2}\right)=\{1.07306,-0.0736\}=$ spectrum of $\gamma_{D^{N}}\left(G_{2}\right)$

Eigen values of $\sigma_{D^{N}}\left(G_{2}\right)=\{1.0281,-0.0281\}=$ spectrum of $\sigma_{D^{N}}\left(G_{2}\right)$

Dominating energy of neutrosophic graph

$\mathrm{G}=(\mathrm{V}, \mathrm{E})=\left[\sum_{\lambda_{\mathrm{i}} \in \mathrm{X}}\left|\lambda_{\mathrm{i}}\right|, \sum_{\delta_{\mathrm{i}} \in \mathrm{Y}}\left|\delta_{\mathrm{i}}\right|, \sum_{\rho_{\mathrm{i}} \in \mathrm{Z}}\left|\rho_{\mathrm{i}}\right|\right]=[0.0183,1.1466,1.0562]$

Now we find the dominating energy of union of neutrosophic graph $G_{1} \cup G_{2}$

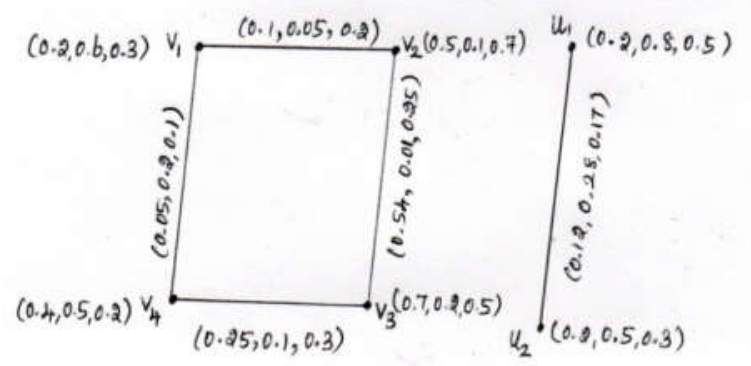

Fig.6: $G_{1} \cup D_{2}$

$$
\begin{gathered}
\mu_{1}\left(v_{1}\right)=\min \left[\mu\left(v_{1} v_{2}\right), \mu\left(v_{1} v_{4}\right)\right]=\min [0.1,0.05]=0.05 \\
\mu_{1}\left(v_{2}\right)=\min \left[\mu\left(v_{2} v_{1}\right), \mu\left(v_{2} v_{3}\right)\right]=\min [0.54,0.1]=0.1 \\
\mu_{1}\left(v_{3}\right)=\min \left[\mu\left(v_{3} v_{2}\right), \mu\left(v_{3} v_{4}\right)\right]=\min [0.54,0.25]=0.25 \\
\mu_{1}\left(v_{4}\right)=\min \left[\mu\left(v_{4} v_{1}\right), \mu\left(v_{4} v_{3}\right)\right]=\min [0.05,0.25]=0.05 \\
\mu_{1}\left(u_{1}\right)=\min \left[\mu\left(u_{1} u_{2}\right]=\min [0.12]=0.12\right. \\
\mu_{1}\left(u_{2}\right)=\min \left[\mu\left(v_{2} u_{1}\right)\right]=\min [0.12]=0.12 \\
\gamma_{1}\left(v_{1}\right)=\max \left[\gamma\left(v_{1} v_{2}\right), \gamma\left(v_{1} v_{4}\right)\right]=\max [0.05,0.2]=0.2 \\
\gamma_{1}\left(v_{2}\right)=\max \left[\gamma\left(v_{2} v_{1}\right), \gamma\left(v_{2} v_{3}\right)\right]=\max [0.05,0.01]=0.05 \\
\gamma_{1}\left(v_{3}\right)=\max \left[\gamma\left(v_{3} v_{2}\right), \gamma\left(v_{3} v_{4}\right)\right]=\max [0.01,0.1]=0.1 \\
\gamma_{1}\left(v_{4}\right)=\max \left[\gamma\left(v_{4} v_{1}\right), \gamma\left(v_{4} v_{3}\right)\right]=\max [0.2,0.1]=0.2 \\
\gamma_{1}\left(u_{1}\right)=\max \left[\gamma\left(u_{1} u_{2}\right)\right]=\max [0.28]=0.28 \\
\gamma_{1}\left(u_{2}\right)=\max \left[\gamma\left(u_{2} u_{1}\right)\right]=\max [0.28]=0.28 \\
\sigma_{1}\left(v_{1}\right)=\max \left[\sigma\left(v_{1} v_{2}\right), \sigma\left(v_{1} v_{4}\right)\right]=\max [0.2,0.1]=0.2
\end{gathered}
$$




$$
\begin{gathered}
\sigma_{1}\left(\mathrm{v}_{2}\right)=\max \left[\sigma\left(\mathrm{v}_{2} \mathrm{v}_{1}\right), \sigma\left(\mathrm{v}_{2} \mathrm{v}_{3}\right)\right]=\max [0.2,0.25]=0.25 \\
\sigma_{1}\left(\mathrm{v}_{3}\right)=\max \left[\sigma\left(\mathrm{v}_{3} \mathrm{v}_{2}\right), \sigma\left(\mathrm{v}_{3} \mathrm{v}_{4}\right)\right]=\max [0.25,0.3]=0.3 \\
\sigma_{1}\left(\mathrm{v}_{4}\right)=\max \left[\sigma\left(\mathrm{v}_{4} \mathrm{v}_{1}\right), \sigma\left(\mathrm{v}_{4} \mathrm{v}_{3}\right)\right]=\max [0.1,0.3]=0.3 \\
\sigma_{1}\left(\mathrm{u}_{1}\right)=\max \left[\sigma\left(\mathrm{u}_{1} \mathrm{u}_{2}\right)\right]=\max [0.17]=0.17 \\
\sigma_{1}\left(\mathrm{u}_{2}\right)=\max \left[\sigma\left(\mathrm{u}_{2} \mathrm{u}_{1}\right)\right]=\max [0.17]=0.17
\end{gathered}
$$

Here, $\mathrm{v}_{1}$ dominates $\mathrm{v}_{4}$ because

$$
\begin{gathered}
\mu\left(\mathrm{v}_{1} \mathrm{v}_{4}\right) \leq \mu_{1}\left(\mathrm{v}_{1}\right) \wedge \mu_{1}\left(\mathrm{v}_{4}\right) 0.05 \leq 0.05 \wedge 0.05 \\
\gamma\left(\mathrm{v}_{1} \mathrm{v}_{4}\right) \leq \gamma\left(\mathrm{v}_{1}\right) \wedge \gamma\left(\mathrm{v}_{4}\right) 0.2 \leq 0.2 \wedge 0.2 \\
\sigma\left(\mathrm{v}_{1} \mathrm{v}_{4}\right) \leq \sigma\left(\mathrm{v}_{1}\right) \wedge \sigma\left(\mathrm{v}_{4}\right) 0.05 \leq 0.2 \wedge 0.3
\end{gathered}
$$

Here, $\mathrm{u}_{1}$ dominates $\mathrm{u}_{2}$ because

$$
\begin{aligned}
& \mu\left(\mathrm{u}_{1} \mathrm{v}_{2}\right) \leq \mu_{1}\left(\mathrm{u}_{1}\right) \wedge \mu_{1}\left(\mathrm{u}_{2}\right) 0.12 \leq 0.12 \wedge 0.12 \\
& \gamma\left(\mathrm{u}_{1} \mathrm{u}_{2}\right) \leq \gamma_{1}\left(\mathrm{u}_{1}\right) \wedge \gamma_{1}\left(\mathrm{u}_{2}\right) 0.28 \leq 0.28 \wedge 0.28 \\
& \sigma\left(\mathrm{u}_{1} \mathrm{u}_{2}\right) \leq \sigma_{1}\left(\mathrm{u}_{1}\right) \wedge \sigma_{1}\left(\mathrm{u}_{2}\right) 0.17 \leq 0.17 \wedge 0.17
\end{aligned}
$$

$\mathrm{V}=\left\{\mathrm{v}_{1}, \mathrm{v}_{2}, \mathrm{v}_{3}, \mathrm{v}_{4}, \mathrm{u}_{1}, \mathrm{u}_{2}\right\}, \mathrm{D}^{\mathrm{N}}=\left\{\mathrm{v}_{1}, \mathrm{u}_{1}\right\}$ and $\mathrm{V}-\mathrm{D}^{\mathrm{N}}=\left\{\mathrm{v}_{2}, \mathrm{v}_{3}, \mathrm{v}_{4}, \mathrm{u}_{2}\right\}$

$\left|D^{N}\right|=2=$ sum of dominating elements

$$
\mathrm{D}^{\mathrm{N}}\left(\mathrm{G}_{1} \cup \mathrm{G}_{2}\right)=
$$

$\left.\begin{array}{llllll}(1,1,1) & (0.1,0.05,0.2) & (0,0,0) & (0.05,0.2,0.1) & (0,0,0) & (0,0,0) \\ (0.1,0.05,0.2) & (0,0,0) & (0.54,0.01,0.25) & (0,0,0) & (0,0,0) & (0,0,0) \\ (0,0,0,) & (0.54,0.01,0.25) & (0,0,0) & (0.25,0.1,0.3) & (0,0,0) & (0,0,0) \\ (0.05,0.2,0.1) & (0,0,0) & (0.25,0.1,0.3) & (0,0,0) & (0,0,0) & (0.12,0.28,0.17) \\ (0,0,0,) & (0,0,0) & (0,0,0) & (0,0,0) & (1,1,1) & (0,0,0) \\ (0,0,0) & (0,0,0) & (0,0,0) & (0,0,0) & (0.12,0.28,0.17) & (0,0,0)\end{array}\right]$

where

$\mu_{\mathrm{D}^{\mathrm{N}}}\left(\mathrm{G}_{1} \cup \mathrm{G}_{2}\right)=\left[\begin{array}{llllll}1 & 0.1 & 0 & 0.05 & 0 & 0 \\ 0.1 & 0 & 0.54 & 0 & 0 & 0 \\ 0 & 0.54 & 0 & 0.25 & 0 & 0 \\ 0.05 & 0 & 0.25 & 0 & 0 & 0 \\ 0 & 0 & 0 & 0 & 1 & 0.12 \\ 0 & 0 & 0 & 0 & 0.12 & 0\end{array}\right],\left(\mathrm{G}_{1} \cup \mathrm{G}_{2}\right)=\left[\begin{array}{llllll}1 & 0.05 & 0 & 0.2 & 0 & 0 \\ 0.05 & 0 & 0.01 & 0 & 0 & 0 \\ 0 & 0.54 & 0 & 0.25 & 0 & 0 \\ 0.05 & 0 & 0.25 & 0 & 0 & 0 \\ 0 & 0 & 0 & 0 & 1 & 0.28 \\ 0 & 0 & 0 & 0 & 0.28 & 0\end{array}\right]$ and

$$
\sigma_{\mathrm{D}^{\mathrm{N}}}\left(\mathrm{G}_{1} \cup \mathrm{G}_{2}\right)=\left[\begin{array}{llllll}
1 & 0.2 & 0 & 0.1 & 0 & 0 \\
0.2 & 0 & 0.25 & 0 & 0 & 0 \\
0 & 0.25 & 0 & 0.3 & 0 & 0 \\
0.1 & 0 & 0.3 & 0 & 0 & 0 \\
0 & 0 & 0 & 0 & 1 & 0.17 \\
0 & 0 & 0 & 0 & 0.17 & 0
\end{array}\right]
$$


Eigen values of $\mu_{D^{N}}\left(G_{1} \cup G_{2}\right)=\{1.0186,-0.5989,0.5803,0,1.0141,-0.0141\}=$ spectrum of $\mu_{D^{N}}\left(G_{1} \cup G_{2}\right)$

Eigen values of $\gamma_{D^{N}}\left(G_{1} \cup G_{2}\right)=\{1.0143,0.0173,-0.2718,0.2401,1.0730,-0.0730\}=$ spectrum of $\gamma_{D^{N}}\left(G_{1} \cup G_{2}\right)$

Eigen values of $\sigma_{D^{N}}\left(G_{1} \cup G_{2}\right)=\{1.0537,-0.4059,0.3601,-0.0079,1.028,-0.0281\}=$ spectrum of $\sigma_{D^{N}}\left(G_{1} \cup G_{2}\right)$

Dominating energy of union of neutrosophic graph

$G=(V, E)=\left[\sum_{\lambda_{\mathrm{i}} \in \mathrm{X}}\left|\lambda_{\mathrm{i}}\right|, \sum_{\delta_{\mathrm{i}} \in \mathrm{Y}}\left|\delta_{\mathrm{i}}\right|, \sum_{\rho_{\mathrm{i}} \in \mathrm{Z}}\left|\rho_{\mathrm{i}}\right|\right]=[3.226,2.6895,2.8897]$

\subsection{Dominating energy in join of neutrosophic graph}

The join of two neutrosophic graph

$G=G_{1}+G_{2}=\left\langle V_{1} \cup V_{2}, E_{1} \cup E_{2}\right\rangle$ defined by

$\left(\mu_{1}+\mu_{1 \prime}\right)(v)=\left(\mu_{1} \cup \mu_{1 \prime}\right)(v)$ if $v \in V_{1} \cup V_{2}$

$\left(\gamma_{1}+\gamma_{1},\right)(v)=\left(\gamma_{1} \cup \gamma_{1},\right)(v)$ if $v \in V_{1} \cup V_{2}$

$\left(\sigma_{1}+\sigma_{1}\right)(v)=\left(\sigma_{1} \cup \sigma_{1}^{\prime}\right)(v)$ if $v \in V_{1} \cup V_{2}$

$\left(\mu_{2}+\mu_{2 \prime}\right)\left(v_{i} v_{j}\right)=\left(\mu_{2} \cup \mu_{2 \prime}\right)\left(v_{i} v_{j}\right)$ if $v_{i} v_{j} \in E_{1} \cup E_{2}$

Now we find the dominating energy of join of neutrosophic graph $G(V, E)$ :

Consider a dominating neutrosophic graph $G=\left(V, E, \mu, \gamma, \sigma, \mu_{1}, \gamma_{1}, \sigma_{1}\right)$ where $V=\left\{v_{1}, v_{2}, v_{3}, v_{4}\right\}$ and $\mu_{1}, \gamma_{1}, \sigma_{1}$ are given by $\mu_{1}: \mathrm{V} \rightarrow[0,1], \gamma_{1}: \mathrm{V} \rightarrow[0,1]$ and $\sigma_{1}: \mathrm{V} \rightarrow[0,1]$ where

\section{$(0.27,0.18,0.23)$}

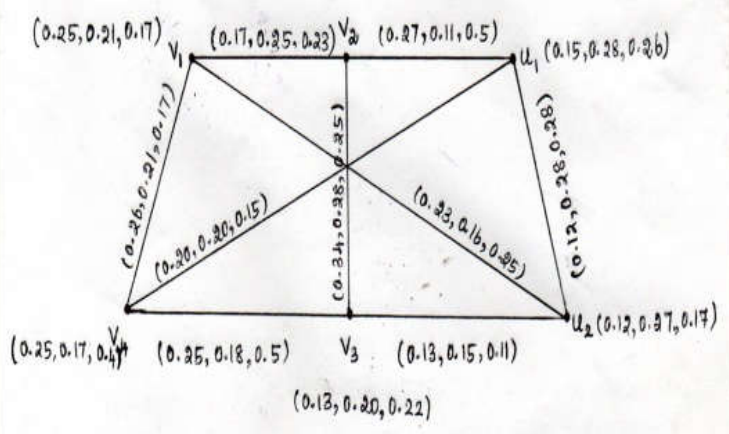

Fig.7: $G=G_{1}+G_{2}$

$$
\begin{gathered}
\mu_{1}\left(v_{1}\right)=\min \left[\mu\left(v_{1} v_{2}\right), \mu\left(v_{1} u_{2}\right), \mu\left(v_{1} v_{4}\right)\right]=\min [0.17,0.23,0.26]=0.17 \\
\mu_{1}\left(v_{2}\right)=\min \left[\mu\left(v_{2} v_{1}\right), \mu\left(v_{2} u_{1}\right), \mu\left(v_{2} v_{3}\right)\right]=\min [0.17,0.27,0.34]=0.17 \\
\mu_{1}\left(v_{3}\right)=\min \left[\mu\left(v_{3} v_{2}\right), \mu\left(v_{3} v_{4}\right), \mu\left(v_{3} u_{2}\right)\right]=\min [0.34,0.24,0.13]=0.13 \\
\mu_{1}\left(v_{4}\right)=\min \left[\mu\left(v_{4} v_{1}\right), \mu\left(v_{4} u_{1}\right), \mu\left(v_{4} v_{3}\right)\right]=\min [0.26,0.20,0.25] \\
\mu_{1}\left(u_{1}\right)=\min \left[\mu\left(u_{1} v_{2}\right), \mu\left(u_{1} v_{4}\right), \mu\left(u_{1} u_{2}\right)\right]=\min [0.27,0.20,0.12]=0.12
\end{gathered}
$$




$$
\begin{aligned}
& \mu_{1}\left(\mathrm{u}_{2}\right)=\min \left[\mu\left(\mathrm{u}_{2} \mathrm{u}_{1}\right), \mu\left(\mathrm{u}_{2} \mathrm{v}_{1}\right), \mu\left(\mathrm{u}_{2} \mathrm{v}_{3}\right)\right]=\min [0.12,0.23,0.13]=0.12 \\
& \gamma_{1}\left(\mathrm{v}_{1}\right)=\max \left[\gamma\left(\mathrm{v}_{1} \mathrm{v}_{2}\right), \gamma\left(\mathrm{v}_{1} \mathrm{u}_{2}\right), \gamma\left(\mathrm{v}_{1} \mathrm{v}_{4}\right)\right]=\max [0.25,0.16,0.21]=0.25 \\
& \gamma_{1}\left(\mathrm{v}_{2}\right)=\max \left[\gamma\left(\mathrm{v}_{2} \mathrm{v}_{1}\right), \gamma\left(\mathrm{v}_{2} \mathrm{u}_{1}\right), \gamma\left(\mathrm{v}_{2} \mathrm{v}_{3}\right)\right]=\max [0.25,0.11,0.28]=0.28 \\
& \gamma_{1}\left(\mathrm{v}_{3}\right)=\max \left[\gamma\left(\mathrm{v}_{3} \mathrm{v}_{2}\right), \gamma\left(\mathrm{v}_{3} \mathrm{v}_{4}, \gamma\left(\mathrm{v}_{3} \mathrm{u}_{2}\right)\right]=\max [0.28,0.18,0.15]=0.28\right. \\
& \gamma_{1}\left(\mathrm{v}_{4}\right)=\max \left[\gamma\left(\mathrm{v}_{4} \mathrm{v}_{1}\right), \gamma\left(\mathrm{v}_{4} \mathrm{u}_{1}\right), \gamma\left(\mathrm{v}_{4} \mathrm{v}_{3}\right)\right]=\max [0.21,0.20,0.18]=0.21 \\
& \gamma_{1}\left(\mathrm{u}_{1}\right)=\max \left[\gamma\left(\mathrm{u}_{1} \mathrm{v}_{2}\right), \gamma\left(\mathrm{u}_{1} \mathrm{v}_{4}\right), \gamma\left(\mathrm{u}_{1} \mathrm{u}_{2}\right)\right]=\max [0.11,0.20,0.28]=0.28 \\
& \gamma_{1}\left(\mathrm{u}_{2}\right)=\max \left[\gamma\left(\mathrm{u}_{2} \mathrm{u}_{1}\right), \gamma\left(\mathrm{u}_{2} \mathrm{v}_{1}\right), \gamma\left(\mathrm{u}_{2} \mathrm{v}_{3}\right)\right]=\max [0.28,0.16,0.15]=0.28 \\
& \sigma_{1}\left(\mathrm{v}_{1}\right)=\max \left[\sigma\left(\mathrm{v}_{1} \mathrm{v}_{2}\right), \sigma\left(\mathrm{v}_{1} \mathrm{u}_{2}\right), \sigma\left(\mathrm{v}_{1} \mathrm{v}_{4}\right)\right]=\max [0.23,0.25,0.17]=0.25 \\
& \sigma_{1}\left(\mathrm{v}_{2}\right)=\max \left[\sigma\left(\mathrm{v}_{2} \mathrm{v}_{1}\right), \sigma\left(\mathrm{v}_{2} \mathrm{u}_{1}\right), \sigma\left(\mathrm{v}_{2} \mathrm{v}_{3}\right)\right]=\max [0.23,0.5,0.25]=0.25 \\
& \sigma_{1}\left(\mathrm{v}_{3}\right)=\max \left[\sigma\left(\mathrm{v}_{3} \mathrm{v}_{2}\right), \sigma\left(\mathrm{v}_{3} \mathrm{v}_{4}, \sigma\left(\mathrm{v}_{3} \mathrm{u}_{2}\right)\right]=\max [0.25,0.5,0.11]=0.25\right. \\
& \sigma_{1}\left(\mathrm{v}_{4}\right)=\max \left[\sigma\left(\mathrm{v}_{4} \mathrm{v}_{1}\right), \sigma\left(\mathrm{v}_{4} \mathrm{u}_{1}\right), \sigma\left(\mathrm{v}_{4} \mathrm{v}_{3}\right)\right]=\max [0.17,0.15,0.5]=0.17 \\
& \sigma_{1}\left(\mathrm{u}_{1}\right)=\max \left[\sigma\left(\mathrm{u}_{1} \mathrm{v}_{2}\right), \sigma\left(\mathrm{u}_{1} \mathrm{v}_{4}\right), \sigma\left(\mathrm{u}_{1} \mathrm{u}_{2}\right)\right]=\max [0.5,0.15,0.28]=0.28 \\
& \sigma_{1}\left(\mathrm{u}_{2}\right)=\max \left[\sigma\left(\mathrm{u}_{2} \mathrm{u}_{1}\right), \sigma\left(\mathrm{u}_{2} \mathrm{v}_{1}\right), \sigma\left(\mathrm{u}_{2} \mathrm{v}_{3}\right)\right]=\min [0.28,0.25,0.11]=0.28
\end{aligned}
$$

Here, $\mathrm{v}_{1}$ dominates $\mathrm{v}_{2}$ because

$$
\begin{aligned}
& \mu\left(\mathrm{v}_{1} \mathrm{v}_{2}\right) \leq \mu_{1}\left(\mathrm{v}_{1}\right) \wedge \mu_{1}\left(\mathrm{v}_{2}\right) 0.17 \leq 0.17 \wedge 0.17 \gamma\left(\mathrm{v}_{1} \mathrm{v}_{2}\right) \leq \gamma_{1}\left(\mathrm{v}_{1}\right) \wedge \gamma_{1}\left(\mathrm{v}_{2}\right) 0.25 \leq 0.25 \wedge 0.25 \\
& \mathrm{~V}=\left\{\mathrm{v}_{1}, \mathrm{v}_{2}, \mathrm{v}_{3}, \mathrm{v}_{4}, \mathrm{u}_{1}, \mathrm{u}_{2}\right\}, \mathrm{D}^{\mathrm{N}}=\left\{\mathrm{v}_{1}, \mathrm{u}_{1}\right\} \text { and } \mathrm{V}-\mathrm{D}^{\mathrm{N}}=\left\{\mathrm{v}_{2}, \mathrm{v}_{3}, \mathrm{v}_{4}, \mathrm{u}_{2}\right\} \\
& \left|\mathrm{D}^{\mathrm{N}}\right|=2=\text { sum of dominating elements }
\end{aligned}
$$

$\left.\begin{array}{llllll}\multicolumn{2}{c}{\mathrm{D}^{\mathrm{N}}(\mathrm{G})=} & & & & \\ (1,1,1) & (0.17,0.25,0.23) & (0,0,0) & (0.26,0.21,0.17) & (0,0,0) & (0,0,0) \\ (0.17,0.25,0.23) & (0,0,0) & (0.36,0.28,0.25) & (0,0,0) & (0.27,0.11,0.5) & (0,0,0) \\ (0,0,0,) & (0.36,0.28,0.25) & (0,0,0) & (0.25,0.18,0.5) & (0,0,0) & (0.13,0.15,0.11) \\ (0.26,0.21,0.17) & (0,0,0) & (0.25,0.18,0.5) & (0,0,0) & (0.20,0.20,0.15) & (0,0,0) \\ (0,0,0,) & (0.27,0.11,0.5) & (0,0,0) & (0.20,0.20,0.15) & (1,1,1) & (0.12,0.28,0.28) \\ (0.17,0.25,0.23) & (0,0,0) & (0.13,0.15,0.11) & (0,0,0) & (0.12,0.28,0.28) & (0,0,0)\end{array}\right]$
where

and

$$
\mu_{\mathrm{D}^{\mathrm{N}}}(\mathrm{G})=\left[\begin{array}{llllll}
1 & 0.17 & 0 & 0.28 & 0 & 0 \\
0.17 & 0 & 0.36 & 0 & 0.27 & 0 \\
0 & 0.36 & 0 & 0.25 & 0 & 0.13 \\
0.26 & 0 & 0.25 & 0 & 0.20 & 0 \\
0 & 0.27 & 0 & 0.20 & 1 & 0.12 \\
0.17 & 0 & 0.13 & 0 & 0.28 & 0
\end{array}\right], \gamma_{\mathrm{D}^{\mathrm{N}}}(\mathrm{G})=\left[\begin{array}{llllll}
1 & 0.25 & 0 & 0.21 & 0 & 0 \\
0.25 & 0 & 0.28 & 0 & 0.11 & 0 \\
0 & 0.28 & 0 & 0.18 & 0 & 0.5 \\
0.21 & 0 & 0.18 & 0 & 0.20 & 0 \\
0 & 0.11 & 0 & 0.20 & 1 & 0.28 \\
0.25 & 0 & 0.15 & 0 & 0.28 & 0
\end{array}\right]
$$




$$
\sigma_{\mathrm{D}^{\mathrm{N}}}(G)=\left[\begin{array}{llllll}
1 & 0.23 & 0 & 0.17 & 0 & 0 \\
0.23 & 0 & 0.25 & 0 & 0.5 & 0 \\
0 & 0.25 & 0 & 0.5 & 0 & 0.11 \\
0.17 & 0 & 0.5 & 0 & 0.5 & 0 \\
0 & 0.5 & 0 & 0.15 & 1 & 0.28 \\
0.23 & 0 & 0.11 & 0 & 0.28 & 0
\end{array}\right]
$$

Eigen values of $\mu_{\mathrm{D}^{\mathrm{N}}}(\mathrm{G})=\{1.2241,1.0118,-0.5402,0.3163,-0.0061+\mathrm{i} 0.0054,-0.0061-\mathrm{i} 0.0054\}=$ spectrum of $\mu_{D^{N}}(G)$

Eigen values of $\gamma_{D^{N}}(G)=\{1.2108,1.0184,-0.5190,0.2687,0.0255,-0.004\}=$ spectrum of $\gamma_{D^{N}}(G)$

Eigen values of $\sigma_{\mathrm{D}^{\mathrm{N}}}(\mathrm{G})=\{1.4069,0.9828,-0.7028,0.4109,-0.1051,0.0072\}=$ spectrum of $\sigma_{\mathrm{D}^{\mathrm{N}}}(\mathrm{G})$

Dominating Energy of join of neutrosophic graph

$\mathrm{G}=(\mathrm{V}, \mathrm{E})=\left[\sum_{\lambda_{\mathrm{i}} \in \mathrm{X}}\left|\lambda_{\mathrm{i}}\right|, \sum_{\delta_{\mathrm{i}} \in \mathrm{Y}}\left|\delta_{\mathrm{i}}\right|, \sum_{\rho_{\mathrm{i}} \in \mathrm{Z}}\left|\rho_{\mathrm{i}}\right|\right]=[0.0121+\mathrm{i} 0.0108,3.0469,3.6157]$

Let $G=\left(V, E, \mu, \gamma, \sigma, \mu_{1}, \gamma_{1}, \sigma_{1}\right)$ be a dominating neutrosophic graph with vertex set $V=\left\{v_{1}, v_{2}, \ldots v_{n}\right\}$, edge set E. Let

$D^{N}=\left\{u_{1}, u_{2}, \ldots u_{n}\right\}$ be a dominating set. If $\lambda_{1}, \lambda_{2}, \ldots \lambda_{n}$ are the eigen values of dominating matrix $\mu_{D^{N}}(G)$ then

(i) $\sum_{\mathrm{i}=1}^{\mathrm{n}} \lambda_{\mathrm{i}}=\left|\mathrm{D}^{\mathrm{N}}\right|$, (ii) $\sum_{\mathrm{i}=1}^{\mathrm{n}} \lambda_{\mathrm{i}}^{2}=\sum_{\mathrm{i}=1}^{\mathrm{n}} \mu_{\mathrm{ii}}^{2}+2 \sum_{1 \leq \mathrm{i}<j \leq n} \mu_{\mathrm{ij}} \mu_{\mathrm{ji}}$

if $\delta_{1}, \delta_{2}, \ldots \delta_{n}$ are the eigen values of dominating matrix $\gamma_{D^{N}}(G)$ then

(iii) $\sum_{\mathrm{i}=1}^{\mathrm{n}} \delta_{\mathrm{i}}=\left|\mathrm{D}^{\mathrm{N}}\right|,(\mathrm{iv}) \sum_{\mathrm{i}=1}^{\mathrm{n}} \delta_{\mathrm{i}}^{2}=\sum_{\mathrm{i}=1}^{\mathrm{n}} \gamma_{\mathrm{ii}}^{2}+\sum_{1 \leq \mathrm{i}<j \leq n} \gamma_{\mathrm{ij}} \gamma_{\mathrm{ji}}$

and if $\rho_{1}, \rho_{2}, \ldots \rho_{\mathrm{n}}$ are the eigen values of dominating matrix $\sigma_{\mathrm{D}^{\mathrm{N}}}(\mathrm{G})$ then $(\mathrm{v}) \sum_{\mathrm{i}=1}^{\mathrm{n}} \rho_{\mathrm{i}}=\left|\mathrm{D}^{\mathrm{N}}\right|$, (vi) $\sum_{\mathrm{i}=1}^{\mathrm{n}} \rho_{\mathrm{i}}^{2}=\sum_{\mathrm{i}=1}^{\mathrm{n}} \sigma_{\mathrm{ii}}^{2}+\sum_{1 \leq \mathrm{i}<j \leq n} \sigma_{\mathrm{ij}} \sigma_{\mathrm{ji}}$

\section{Proof:}

(i) We know that the sum of the eigen values of $\mu_{D^{N}}(G)$ is equal to the trace of $\mu_{D^{N}}(G) \sum_{i=1}^{n} \lambda_{i}=$ $\sum_{\mathrm{i}=1}^{\mathrm{n}} \mu_{\mathrm{ii}}=\left|\mathrm{D}^{\mathrm{N}}\right|$.

(ii) Similarly, the sum of the squares of the eigen values of $\mu_{D^{N}}(G)$ is equal to the trace of $\left(\mu_{D^{N}}(G)\right)^{2}$.

$\sum_{\mathrm{i}=1}^{\mathrm{n}} \lambda_{\mathrm{i}}^{2}=\operatorname{traceof}\left(\mu_{\mathrm{D}^{\mathrm{N}}}(\mathrm{G})\right)^{2}=\mu_{11} \mu_{11}+\mu_{12} \mu_{21}+\mu_{13} \mu_{31}+\ldots \ldots+\mu_{1 \mathrm{n}} \mu_{\mathrm{n} 1}+\mu_{21} \mu_{12}+\mu_{22} \mu_{22}+$
$\mu_{23} \mu_{32}+\ldots \ldots+\mu_{2 n} \mu_{\mathrm{n} 2}+\ldots \ldots \ldots \ldots \ldots \ldots \ldots \ldots \ldots \ldots \ldots \ldots \ldots+\ldots \ldots \ldots+\mu_{\mathrm{n} 1} \mu_{1 \mathrm{n}}+\mu_{\mathrm{n} 2} \mu_{2 \mathrm{n}}+\mu_{\mathrm{n} 3} \mu_{3 \mathrm{n}}+\ldots \ldots+\mu_{\mathrm{nn}} \mu_{\mathrm{nn}}$

$$
\sum_{\mathrm{i}=1}^{\mathrm{n}} \lambda_{\mathrm{i}}^{2}=\sum_{\mathrm{i}=1}^{\mathrm{n}} \mu_{\mathrm{ii}}^{2}+2 \sum_{1 \leq \mathrm{i}<j \leq n} \mu_{\mathrm{ij}} \mu_{\mathrm{ji}}
$$

(iii) We know that the sum of the eigen values of $\gamma_{D^{N}}(G)$ is equal to the trace of $\gamma_{D^{N}}(G) \sum_{i=1}^{n} \delta_{i}=$ $\sum_{\mathrm{i}=1}^{\mathrm{n}} \gamma_{\mathrm{ii}}=\left|\mathrm{D}^{\mathrm{N}}\right|$.

(iv)Similarly, the sum of the squares of the eigen values of $\gamma_{D^{N}}(G)$ is equal to the trace of $\left(\gamma_{D^{N}}(G)\right)^{2}$. 
$\sum_{\mathrm{i}=1}^{\mathrm{n}} \delta_{\mathrm{i}}^{2}=$ trace $\quad$ of $\quad\left(\gamma_{\mathrm{D}^{\mathrm{N}}}(\mathrm{G})\right)^{2}=\quad \gamma_{11} \gamma_{11}+\gamma_{12} \gamma_{21}+\gamma_{13} \gamma_{31}+\ldots .+\gamma_{1 \mathrm{n}} \gamma_{\mathrm{n} 1}+\gamma_{21} \gamma_{12}+\gamma_{22} \gamma_{22}+$ $\gamma_{23} \gamma_{32}+\ldots \ldots+\gamma_{2 n} \gamma_{n 2}+\ldots \ldots \ldots \ldots \ldots \ldots \ldots \ldots \ldots \ldots \ldots+\gamma_{n 1} \gamma_{1 n}+\gamma_{n 2} \gamma_{2 n}+\gamma_{n 3} \gamma_{3 n}+\ldots \ldots+\gamma_{n n} \gamma_{n n}$

$$
\sum_{\mathrm{i}=1}^{\mathrm{n}} \delta_{\mathrm{i}}^{2}=\sum_{\mathrm{i}=1}^{\mathrm{n}} \gamma_{\mathrm{ii}}^{2}+\sum_{1 \leq \mathrm{i}<j \leq n} \gamma_{\mathrm{ij}} \gamma_{\mathrm{ji}}
$$

(v) We know that the sum of the eigen values of $\sigma_{D^{N}}(G)$ is equal to the trace of $\sigma_{D^{N}}(G) \sum_{i=1}^{n} \rho_{i}=$ $\sum_{\mathrm{i}=1}^{\mathrm{n}} \sigma_{\mathrm{ii}}=\left|\mathrm{D}^{\mathrm{N}}\right|$.

(vi)Similarly the sum of the squares of the eigen values of $\sigma_{D^{N}}(G)$ is equal to the trace of $\left(\sigma_{D^{N}}(G)\right)^{2}$.

$\sum_{\mathrm{i}=1}^{\mathrm{n}} \rho_{\mathrm{i}}^{2}=$ trace $\quad$ of $\quad\left(\sigma_{\mathrm{D}^{\mathrm{N}}}(\mathrm{G})\right)^{2}=\sigma_{11} \sigma_{11}+\sigma_{12} \sigma_{21}+\sigma_{13} \sigma_{31}+\ldots .+\sigma_{1 \mathrm{n}} \sigma_{\mathrm{n} 1}+\sigma_{21} \sigma_{12}+\sigma_{22} \sigma_{22}+$ $\sigma_{23} \sigma_{32}+\ldots \ldots+\sigma_{2 n} \sigma_{n 2}+\ldots \ldots \ldots \ldots \ldots \ldots \ldots \ldots \ldots \ldots \ldots \ldots+\sigma_{n 1} \sigma_{1 \mathrm{n}}+\sigma_{\mathrm{n} 2} \sigma_{2 \mathrm{n}}+\sigma_{\mathrm{n} 3} \sigma_{3 \mathrm{n}}+\ldots \ldots+\sigma_{\mathrm{nn}} \sigma_{\mathrm{nn}}$

$\sum_{\mathrm{i}=1}^{\mathrm{n}} \rho_{\mathrm{i}}^{2}=\sum_{\mathrm{i}=1}^{\mathrm{n}} \sigma_{\mathrm{ii}}^{2}+\sum_{1 \leq \mathrm{i}<j \leq n} \sigma_{\mathrm{ij}} \sigma_{\mathrm{ji}} \quad$ Let $\mathrm{G}=\left(\mathrm{V}, \mathrm{E}, \mu, \gamma, \sigma, \mu_{1}, \gamma_{1}, \sigma_{1}\right)$ be a dominating neutrosophic graph with $n$ vertices and $m$ edges. If $\mathrm{D}^{\mathrm{N}}$ is the dominating set then

(i) $\sqrt{\sum_{\mathrm{i}=1}^{\mathrm{n}} \mu_{\mathrm{ii}}^{2}+2 \sum_{1 \leq \mathrm{i}<j \leq n} \mu_{\mathrm{ij}} \mu_{\mathrm{ji}}+\mathrm{n}(\mathrm{n}-1)|\mathrm{A}|^{\frac{2}{n}}} \leq \mathrm{E}\left(\mu_{\mathrm{D}^{\mathrm{N}}} \mathrm{G}\right) \leq \sqrt{\mathrm{n}\left[\sum_{\mathrm{i}=1}^{\mathrm{n}} \mu_{\mathrm{ii}}^{2}+2 \sum_{1 \leq \mathrm{i}<j \leq n} \mu_{\mathrm{ij}} \mu_{\mathrm{ji}}\right]}$ where $|\mathrm{A}|$ is the determinant of $\mu_{D^{N}}(G)$.

(ii) $\sqrt{\sum_{\mathrm{i}=1}^{\mathrm{n}} \gamma_{\mathrm{ii}}^{2}+2 \sum_{1 \leq \mathrm{i}<j \leq n} \gamma_{\mathrm{ij}} \gamma_{\mathrm{ji}}+\mathrm{n}(\mathrm{n}-1)|\mathrm{B}|^{\frac{2}{\mathrm{n}}}} \leq \mathrm{E}\left(\gamma_{\mathrm{D}^{\mathrm{N}}} \mathrm{G}\right) \leq \sqrt{\mathrm{n}\left[\sum_{\mathrm{i}=1}^{\mathrm{n}} \gamma_{\mathrm{ii}}^{2}+2 \sum_{1 \leq \mathrm{i}<j \leq n} \gamma_{\mathrm{ij}} \gamma_{\mathrm{ji}}\right]}$ Where $|B|$ is the determinant of $\gamma_{D^{N}}(G)$.

(iii) $\sqrt{\sum_{\mathrm{i}=1}^{\mathrm{n}} \sigma_{\mathrm{ii}}^{2}+2 \sum_{1 \leq \mathrm{i}<j \leq n} \sigma_{\mathrm{ij}} \sigma_{\mathrm{ji}}+\mathrm{n}(\mathrm{n}-1)|\mathrm{C}|^{\frac{2}{n}}} \leq \mathrm{E}\left(\sigma_{\mathrm{D}^{\mathrm{N}}} \mathrm{G}\right) \leq \sqrt{\mathrm{n}\left[\sum_{\mathrm{i}=1}^{\mathrm{n}} \sigma_{\mathrm{ii}}^{2}+2 \sum_{1 \leq \mathrm{i}<j \leq n} \sigma_{\mathrm{ij}} \sigma_{\mathrm{ji}}\right]}$ Where $|C|$ is the determinant of $\sigma_{D^{N}}(G)$.

Proof: Cauchy Schwarz inequality is $\left[\sum_{\mathrm{i}=1}^{\mathrm{n}} \mathrm{a}_{\mathrm{i}} \mathrm{b}_{\mathrm{i}}\right]^{2} \leq\left[\sum_{\mathrm{i}=1}^{\mathrm{n}} \mathrm{a}_{\mathrm{i}}^{2}\right]\left[\sum_{\mathrm{i}=1}^{\mathrm{n}} \mathrm{b}_{\mathrm{i}}^{2}\right]$

\section{Upper bound:}

$$
\begin{aligned}
& \text { If } a_{i}=1, b_{i}=\left|\lambda_{i}\right| \text { then }\left[\sum_{i=1}^{n}\left|\lambda_{i}\right|\right]^{2} \leq\left[\sum_{i=1}^{n} 1\right]\left[\sum_{i=1}^{n} \lambda_{i}^{2}\right] \\
& \left(E\left(\mu_{D^{N}}(G)\right)\right)^{2} \leq n\left[\sum_{i=1}^{n} \mu_{i i}^{2}+2 \sum_{1 \leq i<j \leq n} \mu_{i j} \mu_{j i}\right] \\
& E\left(\mu_{D^{N}}(G)\right) \leq \sqrt{n\left[\sum_{i=1}^{n} \mu_{i i}^{2}+2 \sum_{1 \leq i<j \leq n} \mu_{i j} \mu_{j i}\right] \ldots \ldots \ldots \ldots . . .}
\end{aligned}
$$

\section{Lower bound:}

$$
\begin{aligned}
\left(E\left(\mu_{D^{N}}(G)\right)\right)^{2}= & {\left[\sum_{i=i}^{n} \mid \lambda_{i}\right]^{2}=\sum_{i=1}^{n}\left|\lambda_{i}\right|^{2}+2 \sum_{1 \leq i<j \leq n}\left|\lambda_{i}\right| \mid \lambda_{j} } \\
& =\left[\sum_{i=1}^{n} \mu_{i i}^{2}+2 \sum_{1 \leq i<j \leq n} \mu_{i j} \mu_{j i}\right]+2 \frac{n(n-1)}{2} A_{1 \leq i<j \leq n}\left\{\left|\lambda_{i}\right|\left|\lambda_{j}\right|\right\}
\end{aligned}
$$

But, $\operatorname{AM}_{1 \leq \mathrm{i}<j \leq n}\left\{\left|\lambda_{\mathrm{i}}\right|\left|\lambda_{\mathrm{j}}\right|\right\} \geq \mathrm{GM}_{1 \leq \mathrm{i}<j \leq n}\left\{\left|\lambda_{\mathrm{i}}\right|\left|\lambda_{\mathrm{j}}\right|\right\}$

Therefore, $\quad E\left(\mu_{D^{N}}(G)\right) \geq \sqrt{\sum_{i=1}^{n} \mu_{i i}^{2}+2 \sum_{1 \leq i<j \leq n} \mu_{i j} \mu_{j i}+n(n-1) G_{1 \leq i<j \leq n}\left\{\left|\lambda_{i}\right|\left|\lambda_{j}\right|\right\}}$ 


$$
\begin{aligned}
& \mathrm{GM}_{1 \leq i<j \leq n}\left\{\left|\lambda_{\mathrm{i}}\right|\left|\lambda_{\mathrm{j}}\right|\right\}=\left[\prod_{1 \leq i<j \leq n}\left|\lambda_{\mathrm{i}}\right|\left|\lambda_{\mathrm{j}}\right|\right]^{\frac{2}{\mathrm{n}(\mathrm{n}-1)}} \\
& =\left[\prod_{\mathrm{i}=1}^{\mathrm{n}}\left|\lambda_{\mathrm{i}}\right|^{\mathrm{n}-1}\right]^{\frac{2}{\mathrm{n}(\mathrm{n}+1)}}=\left[\prod_{\mathrm{i}=1}^{\mathrm{n}}\left|\lambda_{\mathrm{i}}\right|\right]^{\frac{2}{\mathrm{n}}}=|\mathrm{A}|^{\frac{2}{\mathrm{n}}} \mathrm{E}\left(\mu_{D^{N}}(\mathrm{G})\right) \geq
\end{aligned}
$$

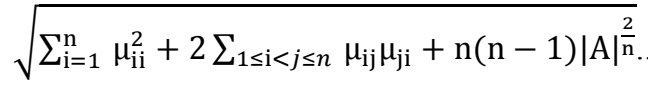

From (1) and (2)

$$
\sqrt{\sum_{\mathrm{i}=1}^{\mathrm{n}} \mu_{\mathrm{ii}}^{2}+2 \sum_{1 \leq \mathrm{i}<j \leq n} \mu_{\mathrm{ij}} \mu_{\mathrm{ji}}+\mathrm{n}(\mathrm{n}-1)|\mathrm{A}|^{\frac{2}{\mathrm{n}}}} \leq \mathrm{E}\left(\mu_{\mathrm{D}^{\mathrm{N}}} \mathrm{G}\right) \leq \sqrt{\mathrm{n}\left[\sum_{\mathrm{i}=1}^{\mathrm{n}} \mu_{\mathrm{ii}}^{2}+2 \sum_{1 \leq \mathrm{i}<j \leq n} \mu_{\mathrm{ij}} \mu_{\mathrm{ji}}\right]}
$$

Similarly, we can prove

$$
\begin{aligned}
& \sqrt{\sum_{\mathrm{i}=1}^{\mathrm{n}} \gamma_{\mathrm{ii}}^{2}+2 \sum_{1 \leq \mathrm{i}<j \leq n} \gamma_{\mathrm{ij}} \gamma_{\mathrm{ji}}+\mathrm{n}(\mathrm{n}-1)|\mathrm{B}|^{\frac{2}{n}}} \leq \mathrm{E}\left(\gamma_{\mathrm{D}^{\mathrm{N}}} \mathrm{G}\right) \leq \sqrt{\mathrm{n}\left[\sum_{\mathrm{i}=1}^{\mathrm{n}} \gamma_{\mathrm{ii}}^{2}+2 \sum_{1 \leq \mathrm{i}<j \leq n} \gamma_{\mathrm{ij}} \gamma_{\mathrm{ji}}\right]} \text { and } \\
& \sqrt{\sum_{\mathrm{i}=1}^{\mathrm{n}} \sigma_{\mathrm{ii}}^{2}+2 \sum_{1 \leq \mathrm{i}<j \leq n} \sigma_{\mathrm{ij}} \sigma_{\mathrm{ji}}+\mathrm{n}(\mathrm{n}-1)|\mathrm{C}|^{\frac{2}{n}}} \leq \mathrm{E}\left(\sigma_{\mathrm{D}^{\mathrm{N}}} \mathrm{G}\right) \leq \sqrt{\mathrm{n}\left[\sum_{\mathrm{i}=1}^{\mathrm{n}} \sigma_{\mathrm{ii}}^{2}+2 \sum_{1 \leq \mathrm{i}<j \leq n} \sigma_{\mathrm{ij}} \sigma_{\mathrm{ji}}\right]}
\end{aligned}
$$

Let $G=(V, E, \mu, \gamma, \sigma)$ be an neutrosophic graph and let $A(G)=(\mu(G), \gamma(G), \sigma(G))$ be an neutrosophic graph adjacency matrix of $G$. Let $G_{1}=\left(V, E, \mu, \gamma, \sigma, \mu_{1}, \gamma_{1}, \sigma_{1}\right)$ be the dominating neutrosophic graph of $G$ and let $D(G)=\left(\mu_{D^{N}}(G), \gamma_{D^{N}}(G), \sigma_{D^{N}}(G)\right)$ be the dominating neutrosophic adjacency matrix of $G_{1}$. Then

$$
\text { (i) }\left(\mathrm{E}\left(\mu_{\mathrm{D}^{\mathrm{N}}}(\mathrm{G})\right)\right)^{2} \leq \mathrm{n}\left[\sum_{\mathrm{i}=1}^{\mathrm{n}} \mu_{\mathrm{ii}}^{2}+(\mathrm{E}(\mu(\mathrm{G})))^{2}\right](\mathrm{ii})\left(\mathrm{E}\left(\gamma_{\mathrm{D}^{\mathrm{N}}}(\mathrm{G})\right)\right)^{2} \leq \mathrm{n}\left[\sum_{\mathrm{i}=1}^{\mathrm{n}} \gamma_{\mathrm{ii}}^{2}+\right.
$$

$\left.(\mathrm{E}(\gamma(\mathrm{G})))^{2}\right](\mathrm{iii})\left(\mathrm{E}\left(\sigma_{\mathrm{D}^{\mathrm{N}}}(\mathrm{G})\right)\right)^{2} \leq \mathrm{n}\left[\sum_{\mathrm{i}=1}^{\mathrm{n}} \sigma_{\mathrm{ii}}^{2}+(\mathrm{E}(\sigma(\mathrm{G})))^{2}\right]$

\section{Proof:}

$$
\begin{gathered}
(\mathrm{E}(\mu(\mathrm{G})))^{2} \geq 2 \sum_{1 \leq \mathrm{i}<j \leq n} \mu_{\mathrm{ij}} \mu_{\mathrm{ji}}+\mathrm{n}(\mathrm{n}-1)|\mathrm{A}|^{\frac{2}{\mathrm{n}}} \geq 2 \sum_{1 \leq \mathrm{i}<j \leq n} \mu_{\mathrm{ij}} \mu_{\mathrm{ji}}(\mathrm{i} . \mathrm{e}) 2 \sum_{1 \leq \mathrm{i}<j \leq n} \mu_{\mathrm{ij}} \mu_{\mathrm{ji}} \leq(\mathrm{E}(\mu(\mathrm{G})))^{2} \\
\quad \text { Now }\left(\mathrm{E}\left(\mu_{\mathrm{D}^{\mathrm{N}}}(\mathrm{G})\right)\right)^{2} \leq \mathrm{n} \sum_{\mathrm{i}=1}^{\mathrm{n}} \mu_{\mathrm{ii}}^{2}+2 \mathrm{n} \sum_{1 \leq \mathrm{i}<j \leq n} \mu_{\mathrm{ij}} \mu_{\mathrm{ji}}\left(\mathrm{E}\left(\mu_{\mathrm{D}^{\mathrm{N}}}(\mathrm{G})\right)\right)^{2} \leq \mathrm{n} \sum_{\mathrm{i}=1}^{\mathrm{n}} \mu_{\mathrm{ii}}^{2}+(\mathrm{E}(\mu(\mathrm{G})))^{2}
\end{gathered}
$$

Similarly, we can prove

$$
\begin{aligned}
& (\mathrm{E}(\gamma(\mathrm{G})))^{2} \geq 2 \sum_{1 \leq \mathrm{i}<j \leq n} \gamma_{\mathrm{ij}} \mu_{\mathrm{ji}}+\mathrm{n}(\mathrm{n}-1)|\mathrm{A}|^{\frac{2}{\mathrm{n}}} \geq 2 \sum_{1 \leq \mathrm{i}<j \leq n} \gamma_{\mathrm{ij}} \gamma_{\mathrm{ji}}(\mathrm{i} . \mathrm{e}) 2 \sum_{1 \leq \mathrm{i}<j \leq n} \gamma_{\mathrm{ij}} \gamma_{\mathrm{ji}} \leq(\mathrm{E}(\gamma(\mathrm{G})))^{2} \\
& \quad \text { Now, }\left(\mathrm{E}\left(\gamma_{\mathrm{D}^{\mathrm{N}}}(\mathrm{G})\right)\right)^{2} \leq \mathrm{n} \sum_{\mathrm{i}=1}^{\mathrm{n}} \gamma_{\mathrm{ii}}^{2}+2 \mathrm{n} \sum_{1 \leq \mathrm{i}<j \leq n} \gamma_{\mathrm{ij}} \gamma_{\mathrm{ji}}\left(\mathrm{E}\left(\gamma_{\mathrm{D}^{\mathrm{N}}}(\mathrm{G})\right)\right)^{2} \leq \mathrm{n} \sum_{\mathrm{i}=1}^{\mathrm{n}} \gamma_{\mathrm{ii}}^{2}+(\mathrm{E}(\gamma(\mathrm{G})))^{2} \\
& \quad \text { and } \\
& (\mathrm{E}(\sigma(\mathrm{G})))^{2} \geq 2 \sum_{1 \leq \mathrm{i}<j \leq n} \sigma_{\mathrm{ij}} \mu_{\mathrm{ji}}+\mathrm{n}(\mathrm{n}-1)|\mathrm{A}|^{\frac{2}{n}} \geq 2 \sum_{1 \leq \mathrm{i}<j \leq n} \sigma_{\mathrm{ij}} \sigma_{\mathrm{ji}}(\mathrm{i} . \mathrm{e}) 2 \sum_{1 \leq \mathrm{i}<j \leq n} \sigma_{\mathrm{ij}} \sigma_{\mathrm{ji}} \leq(\mathrm{E}(\sigma(\mathrm{G})))^{2} \\
& \quad \text { Now, }\left(\mathrm{E}\left(\sigma_{\mathrm{D}^{\mathrm{N}}}(\mathrm{G})\right)\right)^{2} \leq \mathrm{n} \sum_{\mathrm{i}=1}^{\mathrm{n}} \sigma_{\mathrm{ii}}^{2}+2 \mathrm{n} \sum_{1 \leq \mathrm{i}<j \leq n} \sigma_{\mathrm{ij}} \sigma_{\mathrm{ji}}\left(\mathrm{E}\left(\sigma_{\mathrm{D}^{\mathrm{N}}}(\mathrm{G})\right)\right)^{2} \leq \mathrm{n} \sum_{\mathrm{i}=1}^{\mathrm{n}} \sigma_{\mathrm{ii}}^{2}+(\mathrm{E}(\sigma(\mathrm{G})))^{2}
\end{aligned}
$$

\section{Conclusion}


The dominating energy of neutrosophic graph is introduced in this proposed research. Dominating energy of a neutrosophic graph, dominating neutrosophic adjacency matrix, eigen values for the dominating energy of neutrosophic graph and complement neutrosophic graphs are defined with examples. Also dominating energy in union and join operations of neutrosophic graph are developed with suitable examples and some theorems in dominating energy of neutrosophic graph are established. These results will be applied in various real life situations in future.

\section{Acknowledgements}

The article has been written with the joint financial support of RUSA-Phase 2.0 grant sanctioned vide letter No.F.24-51/2014-U, Policy (TN Multi-Gen), Dept. of Edn. Govt. of India, Dt. 09.10.2018, UGC-SAP (DRS-I) vide letter No.F.510/8/DRS-I/2016(SAP-I) Dt. 23.08.2016 and DST (FST - level I) 657876570 vide letter No.SR/FIST/MS-I/2018-17 Dt. 20.12.2018.

\section{REFERENCES}

[1] Cockayne.E.J and Hedetnieme, S.T,Towards a Theory of Domination in Graphs, Networks 7, pp.247-261, 1977.

[2] De Jaenisch, C.F.Applications de l'Analyze Mathematique an Jendes Echecs, 1862.

[3] Gutman.I, The Energy of a graph, Ber.Math-Statist.Sekt.Forschungsz.Graz, 103 ,pp.1-22, 1978.

[4] Nagoorgani.A and Chandrasekaran.V.T, Domination in Fuzzy Graph, Advances in Fuzzy sets and systems,1(1),pp.1726, (2006).

[5] Parvathi.R, Karumbigai.M.G. Intuitionistic fuzzy graphs, Notes on Instuitionistic Fuzzy Sets 18(4),pp.48-58, 2012.

[6] Parvathi.R and G.Thamizhendhi, Domination in intuitionistic fuzzy graphs, Fourth Int.Conf.on IFSs, Sofia, 15-16 May 2010,NIFS Vol.16(2),pp.39-49, 2010.

[7] Peide Liu, Qaisar Khan, Jun Ye \& Tahir Mahmood., Group Decision-Making Method Under Hesitant Interval Neutrosophic Uncertain Linguistic Environment, International Journal of Fuzzy Systems, 20(7), pp.2337-2353, 2018.

[8] Praba.B, Chandrasekaran.V.M, Deepa.G, Energy of an Intuitionistic fuzzy graph of Italin journal of pure and applied mathematics-N.32,pp.431-444, 2014.

[9] Qaisar khan, Tahir mahmood and Jun ye., Multiple Attribute Decision-Making Method under Hesitant Single Valued Neutrosophic Uncertain Linguistic Environment, Journal of Inequalities and Special Functions, 8(2), pp.1-17 ,2017.

[10] Qaisar Khan, Nasruddin Hassan and Tahir Mahmood, Neutrosophic Cubic Power Muirhead Mean Operators with Uncertain Data for Multi-Attribute Decision Making, Symmetry,10 , 444, 2018.

[11] Ranjan Kumar, S A Edaltpanah, Sripati Jha , Said Broumi and Arindam Dey, "Neutrosophic Shortest Path Problem ", Neutrosophic sets and systems, Vol.23, pp.5-15, 2018.

[12] Rosenfeld.A, Fuzzy Graphs, in Zadeh.L.A, Fu.K.CS, Tanaka.K and Shimura.M(eds), Fuzzy Sets and their Applications to Cognitive and Decision Process,Academic Press, New York,pp.75-95, 1975.

[13] S Broumi et. al., Single valued Neutrosophic graphs, Journal of New Theory,10, pp.86-101,2016.

[14] S Broumi, Arindam Dey, M Talea, A Bakali, F Smarandache, D Nagarajan, "Shortest path problem using Bellman algorithm under neutrosophic environment", Complex \& Intelligent Systems 5 (4), pp.409-416

[15] S. Broumi, A.Bakali, F. Smarandache, P. K.Singh, and Arindam Dey" Single Valued (2n+1) Sided Polygonal Neutrosophic Numbers and Single Valued (2n) Sided Polygonal Neutrosophic Numbers" Neutrosophic sets and systems, 2019

[16] S Broumi, Arindam Dey, A Bakali, M Talea, F Smarandache, LH Son, D Koley, "Uniform Single Valued Neutrosophic Graphs", Neutrosophic sets and systems, Vol.17, pp.42-49, 2017.

[17] Somasundaram.A and Somasundaram.S., Domination in fuzzy graphs-1, Pattern Recognition Letters,19(9), pp.787$791,1998$.

[18] Tahir Mahmood \& Qaisar Khan, Interval Neutrosophic finite switchboard state machine, Afrika Matematika, 27, pp.1361-1376, 2016.

[19] Vijayraghavan, A.Kaleemulla and S.ShariefBasha, Dominating energy in products of Intuitionistic fuzzy graphs of Global Pharma Technology, Vol.9,Issue 6, pp.64-76.

[20] Zadeh.L.A, Fuzzy sets, Information and Control,8, pp.338-353, 1965.

[21] Mullai.M., Domination in neutrosophic graphs, Neutrosophic Graph Theory and Algorithms, IGI Global Publications, U.S.A., ISBN 9781799813149 , pp.131-147, 2019. 
[22] S. Broumi, L.H. Son, A. Bakali, M. Talea, F. Smarandache, G. Selvachandran, Computing Operational Matrices in Neutrosophic Environments: A Matlab Toolbox, Neutrosophic Sets and Systems, Vol. 18, 58-66, 2017,

[23] S. Broumi, A. Dey, M. Talea, A. Bakali, F. Smarandache, D. Nagarajan, M. Lathamaheswari and R. Kumar, Shortest Path Problem using Bellman Algorithm under Neutrosophic Environment, Complex \& Intelligent Systems, 5, 409-416, 2019, https://doi.org/10.1007/s40747-019-0101-8,

[24] S. Broumi, M.Talea, A. Bakali, F. Smarandache, D.Nagarajan, M. Lathamaheswari and M.Parimala, Shortest path problem in fuzzy, intuitionistic fuzzy and neutrosophic environment: an overview, Complex \& Intelligent Systems, 5, 371-378, 2019. https://doi.org/10.1007/s40747-019-0098-z

[25] S.Broumi,D. Nagarajan, A. Bakali, M. Talea, F. Smarandache, M. Lathamaheswari, The shortest path problem in interval valued trapezoidal and triangular neutrosophic environment, Complex \& Intelligent Systems, 5, 391-402, 2019 .https://doi.org/10.1007/s40747-019-0092-5

[26] S. A. Edalatpanah, A Direct Model for Triangular Neutrosophic Linear Programming, International Journal of Neutrosophic Science, Volume 1, Issue 1, pp. 19-28, 2020

[27] Akbar Rezaei, Florentin Smarandache ,On Neutro-BE-algebras and Anti-BE-algebras, International Journal of Neutrosophic Science, Volume 4, Issue 1, pp. 08-15, 2020

[28] M.A. Ibrahim, A.A.A. Agboola , E.O. Adeleke, S.A. Akinleye, On Neutrosophic Quadruple Hypervector Spaces, International Journal of Neutrosophic Science, Volume 4, Issue 1, PP: 20-35, 2020 